\title{
Phytochemistry, Pharmacology, and Nutraceutical Profile of Carissa Species: An Updated Review
}

\author{
Jyoti Dhatwalia ${ }^{1}$, Amita Kumari ${ }^{1, *}$, Rachna Verma ${ }^{1}{ }^{\oplus}$, Navneet Upadhyay ${ }^{2} \oplus$, Ishita Guleria ${ }^{1}$, Sohan Lal ${ }^{1}$, \\ Shabnam Thakur ${ }^{1}$, Kasahun Gudeta ${ }^{1,3}{ }^{\oplus}$, Vikas Kumar ${ }^{4}{ }^{\oplus}$, Jane C.-J. Chao ${ }^{5,6} \oplus$, Somesh Sharma ${ }^{7}$,

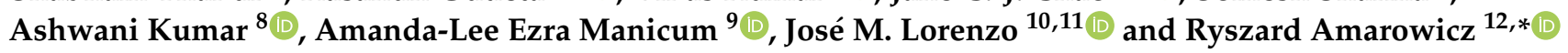

\section{check for} updates

Citation: Dhatwalia, J.; Kumari, A. Verma, R.; Upadhyay, N.; Guleria, I.; Lal, S.; Thakur, S.; Gudeta, K.; Kumar, V.; Chao, J.C.-J.; et al. Phytochemistry, Pharmacology, and Nutraceutical Profile of Carissa Species: An Updated Review. Molecules 2021, 26, 7010. https:// doi.org/10.3390/molecules26227010

Academic Editors: Oksana Sytar and Iryna Smetanska

Received: 1 November 2021

Accepted: 15 November 2021

Published: 20 November 2021

Publisher's Note: MDPI stays neutra with regard to jurisdictional claims in published maps and institutional affiliations.

Copyright: (c) 2021 by the authors. Licensee MDPI, Basel, Switzerland. This article is an open access article distributed under the terms and conditions of the Creative Commons Attribution (CC BY) license (https:// creativecommons.org/licenses/by/ $4.0 /)$.
1 School of Biological and Environmental Sciences, Faculty of Sciences, Shoolini University, Solan 173212, HP, India; dhatwaliajyoti3096@gmail.com (J.D.); rachnac83@gmail.com (R.V.); ishita.thakur93@gmail.com (I.G.); Sohanla148510@gmail.com (S.L.); Shabnamthakur780@gmail.com (S.T.); kasahungudeta40@gmail.com (K.G.)

2 School of Pharmaceutical Sciences, Shoolini University, Solan 173212, HP, India; navneetqa@gmail.com

3 Department of Applied Biology, Adama Science and Technology University, Adama, P.O. Box 1888, Ethiopia

4 University Institute of Biotechnology, Chandigarh University, Gharuan, Mohali, Punjab 140413, India; vikaskmr59@gmail.com

5 School of Nutrition and Health Sciences, College of Nutrition, Taipei Medical University, $250 \mathrm{Wu}-\mathrm{Hsing}$ Street, Taipei 11031, Taiwan; chenjui@tmu.edu.tw

6 Nutrition Research Center, Taipei Medical University Hospital, 252 Wu-Hsing Street, Taipei 11031, Taiwan

7 School of Bioengineering and Food Technology, Shoolini University of Biotechnology and Management, Solan 173229, HP, India; sharmawine@gmail.com

8 Patanjali Herbal Research Department, Patanjali Research Institute, Haridwar, Uttarakhand 249405, India; ashu5157@gmail.com

9 Department of Chemistry, Tshwane University of Technology, Pretoria 0183, South Africa; ManicumAE@tut.ac.za

10 Centro Tecnológico de la Carne de Galicia, Parque Tecnológico de Galicia, 32900 San Cibrao das Viñas, Spain jmlorenzo@ceteca.net

11 Área de Tecnología de los Alimentos, Facultad de Ciencias de Ourense, Universidad de Vigo, 32004 Ourense, Spain

12 Institute of Animal Reproduction and Food Research, Polish Academy of Sciences, 10-748 Olsztyn, Poland

* Correspondence: amitabot@gmail.com (A.K.); r.amarowicz@pan.olsztyn.pl (R.A.)

Abstract: Carissa, a genus of the Apocynaceae family, consists of evergreen species, such as shrubs as well as small trees that are native to Asia, Africa, and Oceania's subtropical and tropical regions. Most of the Carissa species are traditionally used to treat various diseases, such as chest pain, headaches, gonorrhoea, rheumatism, syphilis, oedema, rabies, stomach pain, hepatitis, cardiac diseases, and asthma. The pharmacological studies on Carissa species revealed its antioxidant, antimicrobial, anticancer, cardioprotective, antipyretic, analgesic, wound healing, anticonvulsant, antiarthritic, adaptogenic, anti-inflammatory, and antidiabetic activities, thus validating its use in indigenous medicine systems. The review article summarised the comprehensive literature available, including morphology, indigenous uses, bioactive composition, nutraceutical, and pharmacological activities of Carissa species. A total of 155 research papers were cited in this review article. The Carissa fruits are rich in dietary fibre, lipids, proteins, carbohydrates, vitamin C, and macro- and micro-elements. A total of 121 compounds ( 35 polyphenols (flavonoids and phenolic acids), 30 lignans, 41 terpenoids, 7 steroids, 2 coumarins, and 6 cardiac glycosides) have been extracted from C. spinarum, C. carandas, and C. macrocarpa. Among all chemical constituents, lupeol, carissol, naringin, carisssone, scopoletin, carissaeduloside A, D, J, carandinol, sarhamnoloside, carissanol, olivil, carinol, 3 $\beta$-hydroxyolean11-en-28,13 $\beta$-oilde, ursolic acid, and carissone are the key bioactive constituents responsible for pharmacological activities of genus Carissa. The gathered ethnopharmacological information in the review will help to understand the therapeutic relevance of Carissa as well as paving a way for further exploration in the discovery of novel plant-based drugs. 
Keywords: Carissa; phytochemistry; bioactive compounds; nutraceutical profile; pharmacological activity

\section{Introduction}

Carissa is one of the most important genera in the Apocynaceae family of order Gentianales. It consists of evergreen shrubs or small trees native to the subtropical and tropical regions of Asia, Africa, and Oceania [1]. The genus consists of approximately 85 species, but out of these only 8 have accepted names, whereas other species are either synonyms of these 8 species or assigned to other genera [2]. The species with their accepted names are C. bispinosa (L.) Desf. ex Brenan, C. boiviniana (Baill.) Leeuwenb., C. carandas L., C. haematocarpa (Eckl.) A.DC., C. macrocarpa (Eckl.) A.DC., C. pichoniana Leeuwenb., C. spinarum L., and C. tetramera (Sacleux) Stapf [2]. Traditionally, Carissa plants have been used for the treatment of a variety of diseases, such as headache, syphilis, chest discomfort, gonorrhoea, malaria, arthritis, and rabies, since time immemorial [3-9]. Besides these, they are also used against sickle cell anaemia, ulcers, and worm infections [1]. The fruits of the genus are rich in dietary fibre, lipids, protein, carbohydrates, and macro- and micro-elements, and as a result, they play a crucial role in promoting human health [10].

Previous comprehensive reports on Carissa species are either available only on the traditional uses and phytochemistry [11] or on the research carried out on single species [12,13]. This review provides complete information on various aspects of four Carissa species, e.g., C. carandas, C. macrocarpa [syn. C. grandiflora (E. Mey.) A. Dc], C. bispinosa, and C. spinarum (syn. C. opaca Stapt ex Haines, C. edulis Vahl, C. lanceolata R. Br, and C. congesta Wt.), emphasizing taxonomy, ethnomedicinal and nutraceutical uses, phytochemistry, and pharmacological activities, covering the periods from 1951 to 2021. This information may provide opportunities for researchers around the world to investigate the unexplored species of the genus by isolating new bioactive phytoconstituents.

\subsection{Research Methdology}

The scientific literature was searched through various databases, such as Scopus, Google Scholar, Science Direct, The Plant List, Plant of the World Online, and PubMed. The current review article contains the research carried out on Carissa species in the fields of nutrition, phytochemistry, and pharmacology over the period from 1950 to 2021. The chemical structure of compounds was drawn using Chem draw software. The scientific names of plant species were validated from The Plant List database [2], and their distribution was taken from the Plant of the World Online [14].

\subsection{Distribution of Carissa Species}

Carissa's native range is Africa to Indo-China, Australia to New Caledonia, and has been introduced into the Bahamas, China, Central America, Jamaica, Indonesia, Malaya, Mexico, Nicaragua, the Philippines, Taiwan, Trinidad-Tobago, and the USA [14] (Figure 1).

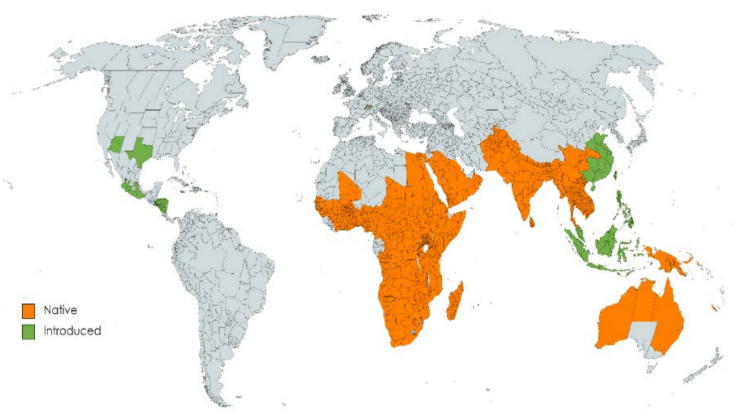

Figure 1. Geographical distribution of Carissa species (Created with mapchart.net). 


\section{General Morphology}

The plants are evergreen, multi-stemmed (C. macrocarpa), sprawling (C. carandas), and dense bush or rambling (C. bispinosa) shrubs (C. spinarum), which grow as a small tree up to 1-5 $\mathrm{m}$ in height. The stem is branched, glabrous, greenish, spiny, and rich in white latex. Leaves are in opposite-decussate arrangement, green, simple, obovate or oblanceolate (C. carandas), ovate or rounded (C. spinarum, C. bispinosa), thick coriaceous (C. macrocarpa), glabrous with obtuse or emarginated (C. carandas), acute or rounded (C. spinarum), apex and acute to cuneate (C. carandas), cuneate or rounded (C. spinarum) base. Flowers begin to bloom between February and April in C. spinarum, March-April in C. carandas, May in C. macrocarpa, and October to March in C. bispinosa. Flowers are small, fragrant, white inside, and pink to red on the outside (C. spinarum), white (C. carandas, C. macrocarpa), jasmine-scented (C. macrocarpa), white or tinged pink (C. bispinosa), glossy, tubular, and 5-lobed. Fruiting takes place throughout the year. The fruits are globose or ellipsoid (C. carandas, C. spinarum), ovoid (C. bispinosa), or pointed (C. macrocarpa) in shape, red (C. macrocarpa, C. bispinosa), dark blue (C. carandas), or red to black (C. spinarum) when fully ripened. Seeds are llipsoid (C. carandas), lanceolate (C. spinarum), and black or brown in colour [8,15-20] (Figure 2).
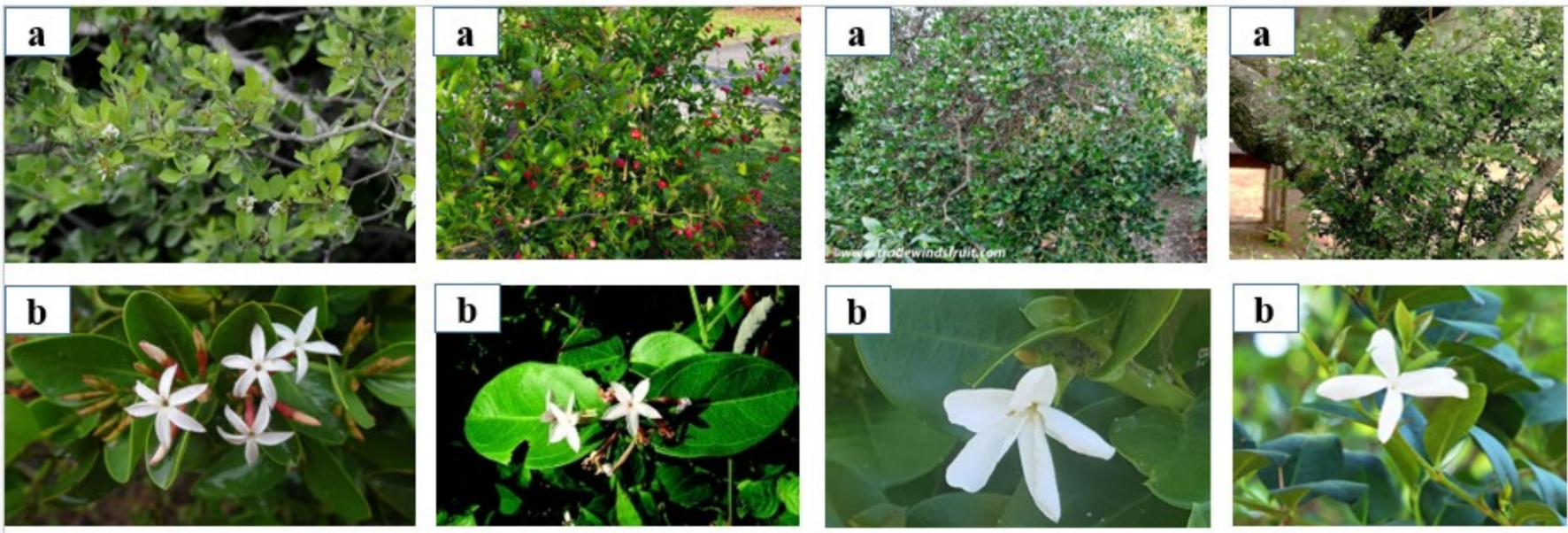

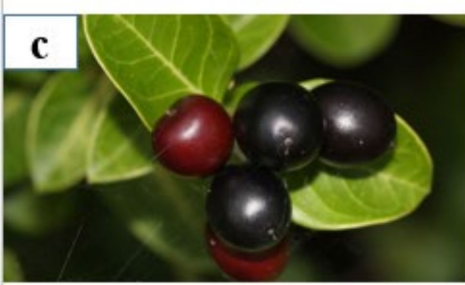

A. Carissa spinarum

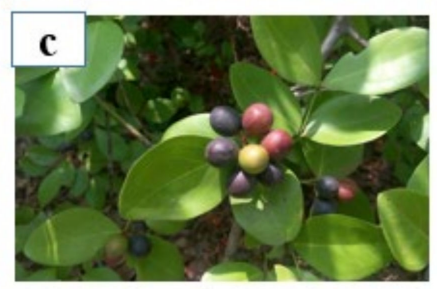

B. Carissa carandas

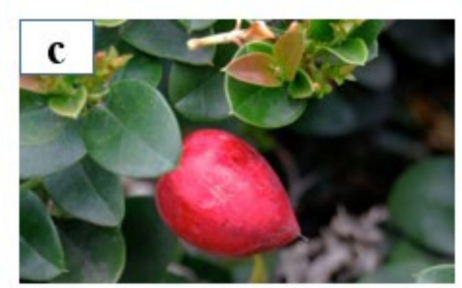

C. Carissa macrocarpa

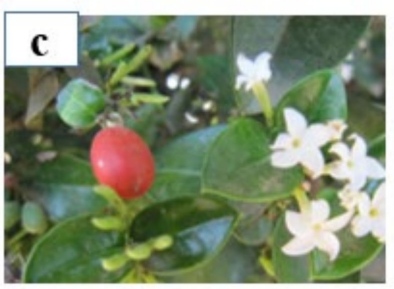

D. Carissa bispinosa

Figure 2. Representative pictures showing morphology of Carissa spinarum (A), C. carandas (B), C. macrocarpa (C), and C. bispinosa (D): a-whole plant; b-flower; c-fruit. Sources: C. spinarum (http://www.plantsoftheworldonline.org, accessed on 10 November 2021); C. carandas plant (http:/ / chengailimfruittrees.blogspot.com, accessed on 10 November 2021); C. carandas flowers (https:/ / indiabiodiversity.org, accessed on 10 November 2021); C. carandas fruits (http:/ / tropical. theferns.info, accessed on 10 November 2021); C. macrocarpa plant and fruit (http://www.tradewindsfruit.com, accessed on 10 November 2021); C. macrocarpa flower (http:/ / www.plantsoftheworldonline.org, accessed on 10 November 2021); C. bispinosa plant (http://natureswow2.blogspot.com, accessed on 10 November 2021); C. bispinosa flowers and fruits (https: / / treesa.org, accessed on 10 November 2021).

\section{Traditional Uses}

Carissa genus is reported with numerous ethnomedicinal uses (Table 1). The plant parts such as leaf, bark, stem, fruit, and roots have found different uses in the management and treatment of diseases such as fever, cold and cough, ulcer, toothache, rheumatism, diarrhoea, epilepsy, anaemia, and cardiovascular diseases [8,15-20]. The whole plant of 
C. spinarum is traditionally used in India, Ethiopia, and other African countries for the remedy of venereal respiratory and gastrointestinal infections [21-31], fever, jaundice, hepatitis, cardiac diseases, asthma [22], gonorrhoea, stomach-ache, chickenpox, wound healing, rabies, and also as an antidote to snake bites [32,33]. The leaves of $C$. spinarum are also used as a mosquito repellent [32]. According to Chandra et al. [22], the consumption of C. spinarum fruits promotes good health and well-being and reduces the risk of chronic disease, whereas roots can provide new leads for antimicrobial drugs [34,35].

Table 1. Ethnomedicinal uses of Carissa species.

\begin{tabular}{|c|c|c|c|}
\hline Carissa Species & Part Used & Indications & References \\
\hline \multirow{3}{*}{ C. spinarum } & Leaves & $\begin{array}{l}\text { Cold and flu, diabetes, malaria, } \\
\text { pneumonia, and } \\
\text { childbirth complications }\end{array}$ & [25] \\
\hline & Fruits & $\begin{array}{l}\text { Constipation, diabetes, heart disease, } \\
\text { and obesity }\end{array}$ & [36] \\
\hline & Roots & $\begin{array}{l}\text { Treat worm-infested wounds } \\
\text { in animal }\end{array}$ & [37] \\
\hline \multirow{3}{*}{ C. spinarum (syn. C. opaca) } & Leaves & Tanning agent, cure fever and asthma & {$[22,38]$} \\
\hline & Fruits & Aphrodisiac & \multirow[b]{2}{*}[38]{} \\
\hline & Whole plant & $\begin{array}{l}\text { Eye disorder, horn injuries, and } \\
\text { maggot wounds in animal }\end{array}$ & \\
\hline \multirow[t]{2}{*}{ C. spinarum (syn. C. lanceolata) } & Bark and twig & $\begin{array}{c}\text { Toothache, respiratory infections, and } \\
\text { cleaning sores }\end{array}$ & \multirow[t]{2}{*}{ [39] } \\
\hline & Leaves & Mosquito repellent & \\
\hline \multirow{3}{*}{ C. spinarum (syn. C. edulis) } & \multirow[t]{2}{*}{ Whole plant } & $\begin{array}{l}\text { Headache, chest complaints, } \\
\text { rheumatism, gonorrhoea, syphilis, } \\
\text { rabies, and epilepsy }\end{array}$ & [3-5] \\
\hline & & Fever, sickle cell anaemia, and hernia & [5] \\
\hline & Leaves and roots & $\begin{array}{l}\text { Anthelmintic, antiscorbutic, } \\
\text { astringent, regulate blood glucose } \\
\text { level, stomach pain, and tooth pain }\end{array}$ & [11] \\
\hline \multirow{6}{*}{ C. carandas } & Roots & $\begin{array}{l}\text { Stomachic, vermifuge, remedy for } \\
\text { itch, and insect repellent }\end{array}$ & {$[20,24]$} \\
\hline & Stem & Strengthens tendons & {$[40]$} \\
\hline & Leaves & $\begin{array}{l}\text { Fevers, earache, diarrhoea, and } \\
\text { diabetic ulcers }\end{array}$ & {$[20,26]$} \\
\hline & Fruits & Anaemia, anti-scorbutic, biliousness & [40] \\
\hline & \multirow{2}{*}{ Whole plant } & $\begin{array}{l}\text { Diarrhoea, anorexia, intermittent } \\
\text { fever, mouth ulcer, sore throat, } \\
\text { syphilitic pain, burning sensation, } \\
\text { scabies, and epilepsy }\end{array}$ & {$[41,42]$} \\
\hline & & $\begin{array}{l}\text { Anthelmintic, appetizer, antipyretic, } \\
\text { stomach disorders, rheumatism, } \\
\text { disease of the brain, biliousness, and } \\
\text { biliary dysfunction }\end{array}$ & {$[43,44]$} \\
\hline \multirow{3}{*}{ C. macrocarpa } & Fruits & $\begin{array}{l}\text { Human immunodeficiency virus } \\
\text { (HIV) and hepatitis }\end{array}$ & {$[8,45]$} \\
\hline & Leaves & Diarrhoea in livestock & {$[8,46]$} \\
\hline & Whole plant & Cough and venereal diseases & [46] \\
\hline C. bispinosa & Roots & Toothache & [47] \\
\hline
\end{tabular}


The role of the $C$. carandas whole plant has been found to be effective in the treatment of liver diseases [48], convulsions [21], asthma, tuberculosis, scabies, and wound healing $[41,42,49]$. Fruits of $C$. macrocarpa have shown their essential role in the remedy of human immunodeficiency virus (HIV) and hepatitis $[8,45]$.

\section{Nutraceutical Profile of Carissa Species}

The Carissa fruits are rich in fibres, lipids, proteins, carbohydrates, and macro- and micro-nutrients, which are essential to build and maintain strong bones and to retain normal functioning of the heart, kidney, muscles, and nerves $[10,46,50]$. The fruits are rich in nutritive compounds that improve taste and also prolong the shelf-life of food products. Ripe fruits are eaten raw and used for making the excellent quality cakes, ice cream, jams, squash, and jelly, which resemble gooseberry in flavour, whereas unripe fruits are used for making chutney, pickles, and candies. C. carandas fruits (ripe) have also been documented to be used as a natural food decolourant cum nutraceutical supplement in the lime sharbat, named "Lalima" [51]. The nutraceutical values of Carissa plants are summarised in Table 2.

Table 2. Nutritional profile of Carissa fruits.

\begin{tabular}{|c|c|c|c|c|c|c|}
\hline \multirow[b]{2}{*}{$\begin{array}{l}\text { Nutritional } \\
\text { Parameters }\end{array}$} & \multicolumn{3}{|c|}{ C. spinarum } & \multirow{2}{*}{$\begin{array}{l}\text { C. carandas } \\
\text { C. carandas }\end{array}$} & \multicolumn{2}{|c|}{ C. macrocarpa } \\
\hline & $\begin{array}{c}\text { C. spinarum } \\
\text { (syn. C. opaca) }\end{array}$ & C. spinarum & $\begin{array}{c}\text { C. spinarum } \\
\text { (syn. C.edulis) }\end{array}$ & & $\begin{array}{l}\text { C. macrocarpa } \\
\text { (syn. } \\
\text { C. grandiflora) }\end{array}$ & C. macrocarpa \\
\hline Country & India, Pakistan & India & Uganda & India & India & Tunisia \\
\hline Moisture (\%) & 76.60 & 81.05 & 84.6 & 88.70 & 78.45 & 78.83 \\
\hline Ash (\%) & $1.25,4.78$ & 2.46 & - & $0.78,0.0018$ & 0.43 & 0.5 \\
\hline Total protein $(\%)$ & $1.30,6.31$ & 2.07 & 0.3 & $2,2.14$ & 0.56 & 0.74 \\
\hline Dietary fibre (\%) & $3.40,13.55$ & - & 2.65 & $1.81,15.64$ & 0.91 & - \\
\hline Crude lipids (\%) & 0.02 & 1.30 & - & 10 & 1.03 & 3.53 \\
\hline Carbohydrates (\%) & $17.39,47$ & 18.66 & - & $67,0.019$ & - & 16.40 \\
\hline Organic matter (\%) & 98.75 & - & - & - & - & - \\
\hline Total sugar (g) & - & - & - & $11.58,0.04$ & 0.12 & - \\
\hline $\begin{array}{l}\text { Non-reducing } \\
\text { sugar (mg) }\end{array}$ & - & - & - & 4.25 & - & - \\
\hline $\begin{array}{l}\text { Reducing } \\
\text { sugar (mg) }\end{array}$ & - & - & - & $41.25 ; 41.5$ & - & - \\
\hline $\begin{array}{l}\text { Ascorbic acid } \\
(\mathrm{mg} / 100 \mathrm{~g})\end{array}$ & & - & 3.24 & 62.93 & 10 & 100 \\
\hline Sodium (mg/100 g) & - & - & 1.79 & - & - & - \\
\hline $\begin{array}{c}\text { Calcium } \\
(\mathrm{mg} / 100 \mathrm{~g})\end{array}$ & 1 & - & 10 & $1.60,2.92$ & - & - \\
\hline $\begin{array}{l}\text { Magnesium } \\
(\mathrm{mg} / 100 \mathrm{~g})\end{array}$ & 8.4 & - & 4 & 5.2 & - & - \\
\hline $\begin{array}{l}\text { Potassium } \\
(\mathrm{mg} / 100 \mathrm{~g})\end{array}$ & 1.98 & - & 198 & - & - & - \\
\hline $\begin{array}{l}\text { Phosphorus } \\
\text { (mg/100 g) }\end{array}$ & 0.24 & - & 24 & 24.15 & - & - \\
\hline Iron $(\mathrm{mg} / 100 \mathrm{~g})$ & 0.56 & - & 0.56 & $3.9,1.088$ & - & - \\
\hline References & {$[10,22]$} & [36] & [51] & {$[49,52-54]$} & [19] & {$[46]$} \\
\hline
\end{tabular}




\section{Phytochemistry}

Genus Carissa is rich in different classes of primary and secondary metabolites, including carbohydrates, lipids, proteins, and phenolics including flavonoids and tannins, terpenoids, coumarins, lignins, glycosides, tannins, and steroids [22,25,44,55-65]. Extensive work has been carried out by researchers to distinguish several compounds from the leaves, stems, roots, and wood of the Carissa species. A total of 93 compounds from C. spinarum (27 polyphenols, 27 lignans, 23 terpenoids, 8 steroids, 2 coumarins, and 6 cardiac glycosides), 28 from C. carandas ( 2 polyphenols, 4 lignans, 20 terpenoids, and 2 steroids), and 11 from C. macrocarpa (6 polyphenols and 5 terpenoids) have been extracted (Figure 3).

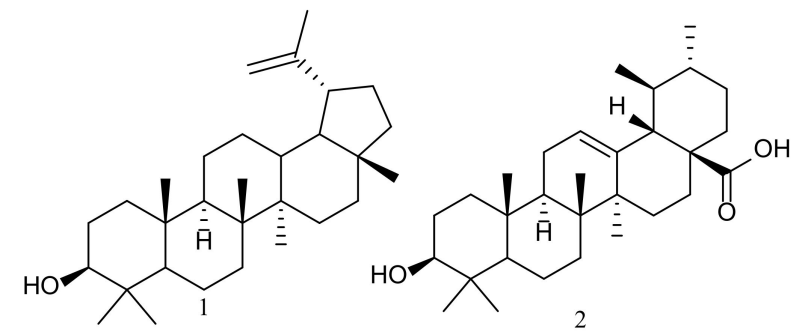<smiles>CC1=C2C[C@H](C(C)(C)O)CC[C@]2(C)CCC1=O</smiles><smiles>COCOCC(C)OCC(C)OCC(C)OCC(C)OCC(C)O</smiles>

10 5<smiles>COc1cc(CC2(O)C(C)COC2O)ccc1O</smiles><smiles>COc1cc(C[C@@H](O)[C@@](O)(CO)Cc2ccc(O)c(OC)c2)ccc1O</smiles>

6

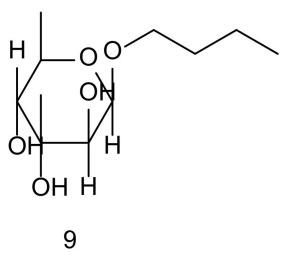<smiles>COc1cc2ccc(=O)oc2cc1O</smiles>

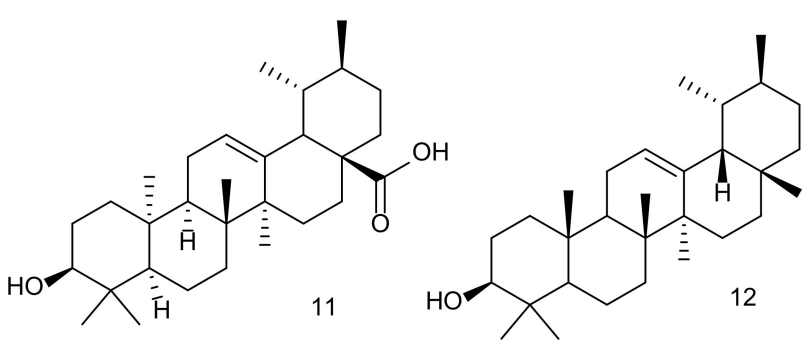<smiles>O=C(CO)c1ccccc1</smiles>

13

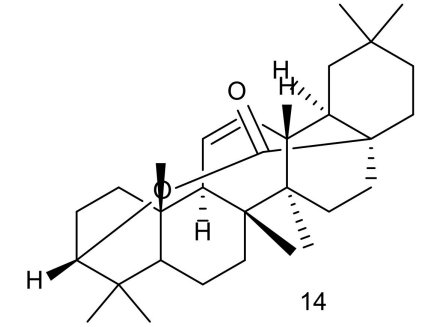<smiles>CC1=C2CC(C(C)(C)O)CC[C@]2(C)C=CC1=O</smiles>

Figure 3. Cont. 

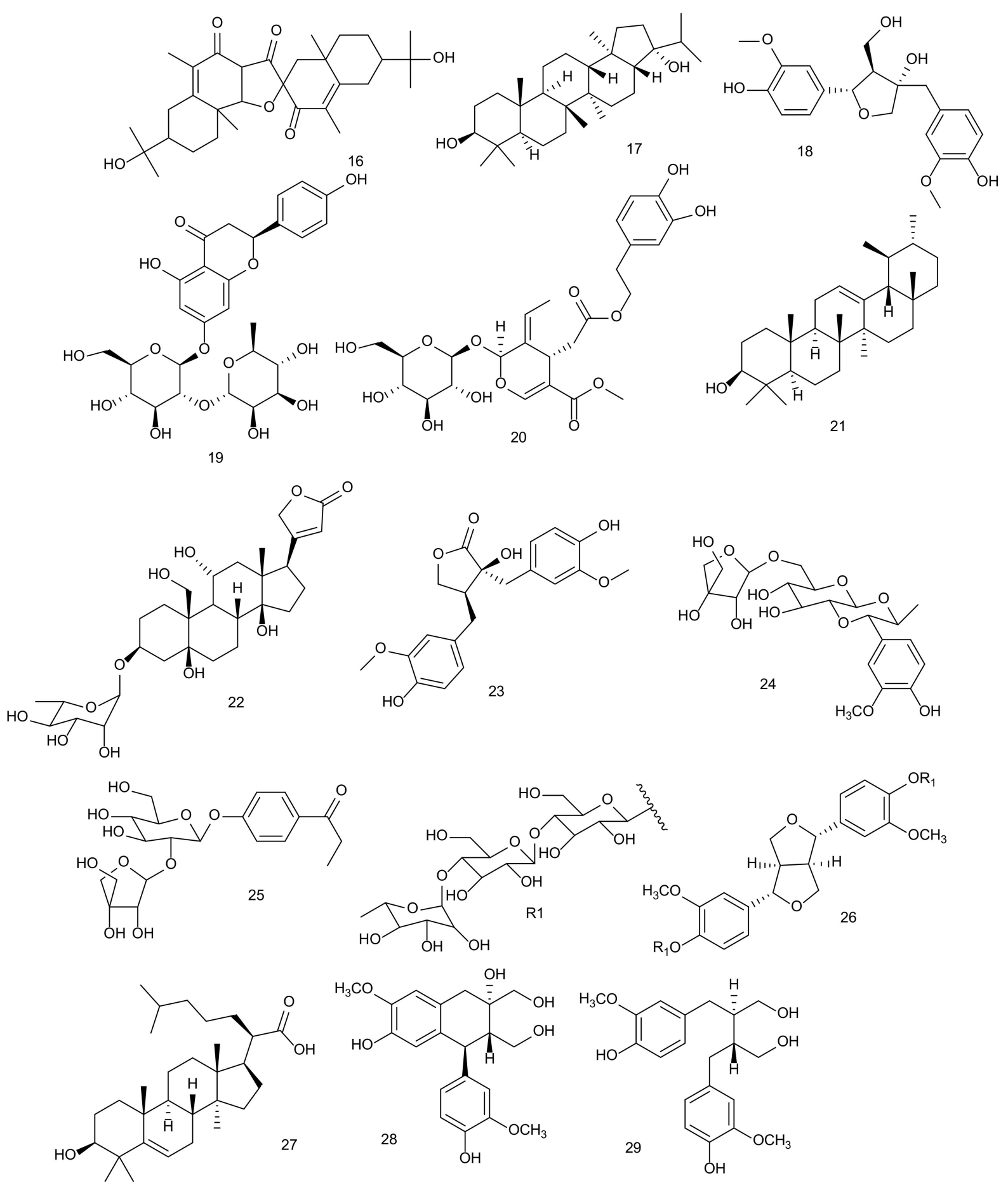

Figure 3. Cont. 


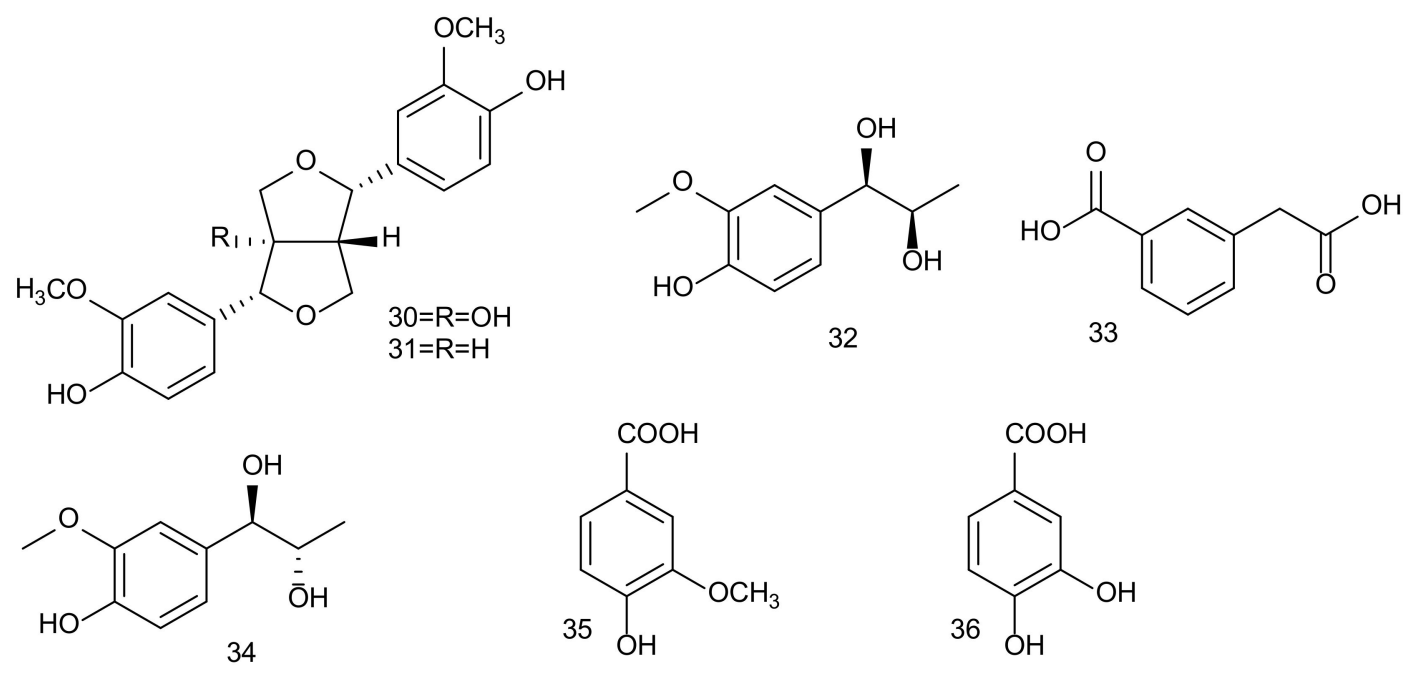<smiles>COc1cc([C@H]2c3c(cc(OC)c(O)c3[O-])C[C@H](CO)[C@H]2CO[C@H]2O[C@H](CO)[C@@H](O)[C@H](O)[C@H]2O)ccc1O</smiles><smiles>COc1cc([C@H]2c3cc(O)c(OC)cc3C[C@H](CO)[C@H]2CO[C@H]2O[C@H](CO)[C@@H](O)[C@H](O)[C@H]2O)ccc1O</smiles><smiles>COc1cc([C@@H]2c3c(cc(OC)c(O)c3O)C[C@H](CO)[C@H]2CO[C@H]2O[C@H](CO)[C@@H](O)[C@H](O)[C@H]2O)cc(OC)c1O</smiles><smiles>COc1cc([C@H]2c3c(cc(OC)c(O)c3O)C[C@@H](CO)[C@H]2CO[C@H]2O[C@H](CO)[C@@H](O)[C@H](O)[C@H]2O)cc(OC)c1O</smiles><smiles>CC(=O)c1ccccc1O[C@@H]1O[C@H](CO[C@H]2OC[C@@H](O)[C@H](O)[C@H]2O)[C@@H](O)[C@H](O)[C@H]1O</smiles>

39<smiles>O=C(O)/C=C/c1ccc(O)cc1</smiles>

Figure 3. Cont. 


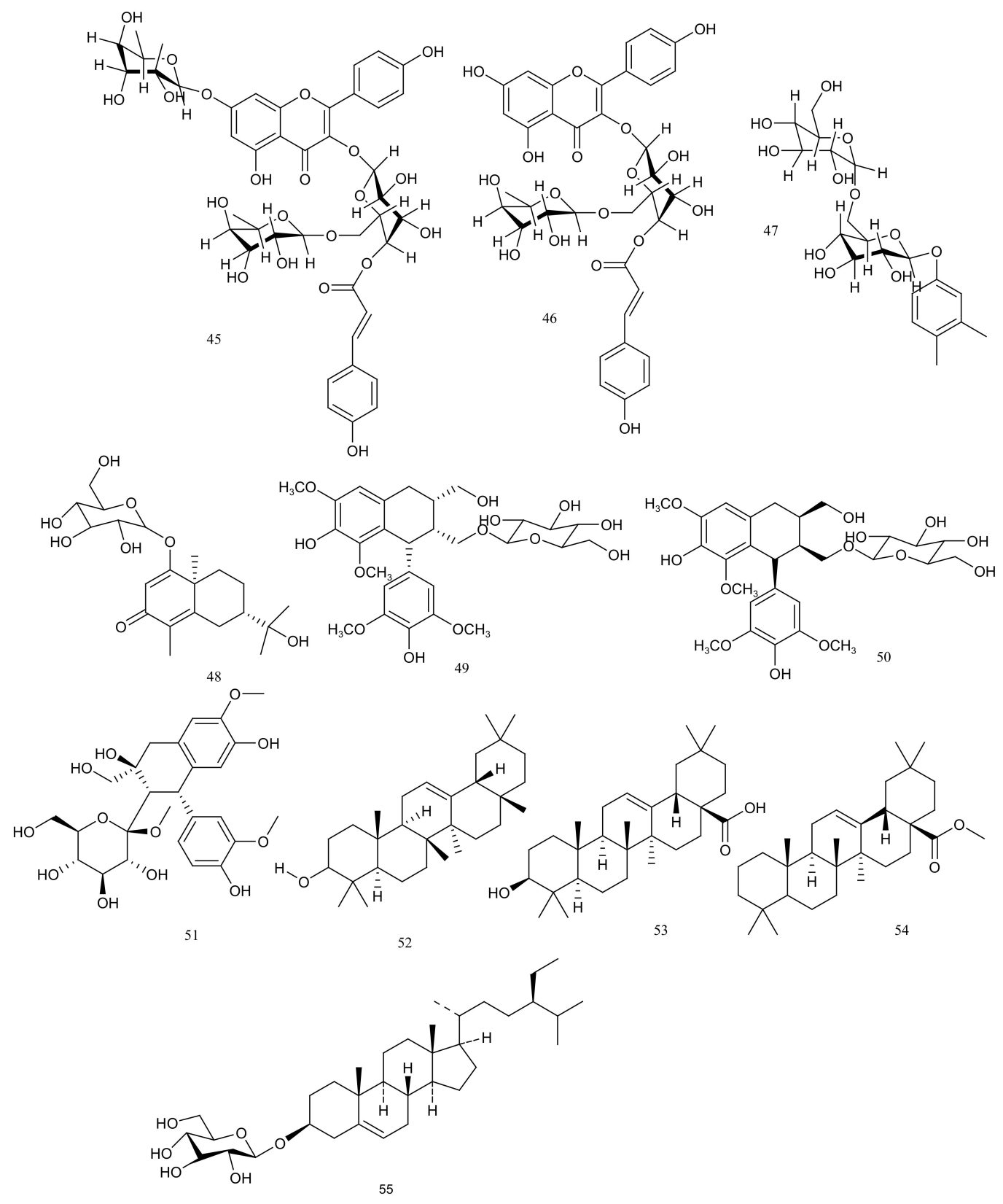

Figure 3. Chemical structure of biologically active isolated compounds of Carissa species: lupeol (1), ursolic acid (2), carissone (3), caredulis,-\{1-[2-(2 hydroxypropoxy) propoxy] propan-2-yloxy\} (4), carissanol (5), carinol (6), $\beta$-sitosterol (7), scopoletin (8), butyl-O- $\alpha$-L-rhamnoside (9), carenone (10), carissic acid (11), carissol (12), 2-hydroxyacetophenone (13), $3 \beta$-hydroxyolean-11-en-28,13ß-olide (14), dehydrocarissone (15), carindone (16), carandinol (17), olivil (18), naringin (19), oleuropein (20), $\alpha$-amyrin (21), sarhamnoloside (22), nortrachelogenin (23), carissaedulosides A (24), carissaedulosides D (25), carissaedulosides J (26), lanost-5-en-3b-ol-21-oic acid (27), (+)-cycloolivil (28), (-)-secoisolariciresinol (29), $\mathrm{R}=\mathrm{OH}((+)-8$ hydroxypinoresinol (30), R=H ((+)-pinoresinol (31), erythro-1-(3-methoxy-4-hydroxy-phenyl)-propan-1,2-diol (32), 3-carboxy methyl-benzoic acid (33), threo-1-(3methoxy-4-hydroxy-phenyl)-propan-1,2-diol (34), vanillic acid (35), protocatechuic acid (36), (6R,7S,8S)-7a-[( $\beta$-D-glucopyranosyl)-oxy]1-methoxy isolariciresinol (37), (+)-isolariciresinol3a-O- $\beta$-D-glucopyranoside (38), (-)lyoniresinol3 $\alpha$-O- $\beta$-D-glucopyranoside (39), (+)-lyoniresinol3 $\alpha-O-\beta$-D-glucopyranoside (40), acetophenone-2-O$\beta x y l o p y r a n o s y l-(1 \rightarrow 6)-O-\beta$-glucopyranoside (41), kaempferol-3-O-robinobioside (42), $p$-coumaric acid (43), salicin (44), kaempferol-3-O- $\alpha$-L-rhamopyranosyl (45), variabiloside E (46), 3,4-dimethylphenol $\beta$-gentiobioside (47), carandoside (48), $(6 S, 7 R, 8 R)-7 a-[(\beta$-glucopyranosyl) oxy]lyoniresinol (49), (6R,7S,8S)-7a-[( $\beta$-glucopyranosyl)oxy]lyoniresinol $(\mathbf{5 0}),[(1 S, 2 S, 3 S)-$ 1,2,3,4-tetrahydro-3,7-dihydroxy-1-(4-hydroxy-3-methoxyphenyl)-3-(hydroxymethyl)-6methoxy-2-naphthalen-yl] methyl $\beta$ D-gluco-pyranoside (51), $\beta$-amyrin (52), methyl oleanate (53), oleanolic acid (54), $\beta$-sitosterol-3-O- $\beta$-D-glucopyranoside (55). 


\subsection{Polyphenols (Phenolic Acids and Flavonoids)}

From the genus Carissa, a total of 35 polyphenols including phenolic acids and flavonoids were isolated. The caffeic acid and carissone were isolated from the roots of $C$. spinarum [66]. Five phenolic compounds (two phenolics (caffeic acid methyl ester and chlorogenic acid-1ethyl ether-1-methyl ester), two flavonols (kaempferol and rutin), and one flavanol glycoside (quercetin-3-O-glucoside-7, $3^{\prime}, 4^{\prime}$-trimethyl ether)) were isolated from the aerial parts of C. edulis (syn. C. spinarum) by Al-Youssaf and Hassan [67]. Likewise, four flavonol glucosides, namely kaempferol 3-O- $\beta$-D glucopyranoside, quercetin-3-O- $\beta$-D glucopyranoside, rhamnetin-3-O- $\beta$-D-glucopyranoside, and isorhamnetin-3-O- $\beta$-D-glucopyranoside, and one derivative of phenolic acid (3-O-acetyl chlorogenic acid) were eluted for the first time from the ethyl acetate fraction of aerial parts of C. edulis (syn. C. spinarum) by AlYoussaf and Hassan [68]. Similarly, from the butanol fraction of aerial parts of C. edulis (syn. C. spinarum), Al-Youssaf and Hassan [69] isolated one flavonol glucoside (isoorhamnetin-3-O$\beta$-D-glucopyranoside-rhamnopyranoside) and two other phenolic compounds (caredulis,1$\{1-[2-(2$ hydroxypropoxy) propoxy]\} propan-2-yloxy (4) and butyl-O- $\alpha$-L-rhamnoside (9)). A flavanone, naringin (19), was isolated from the methanolic leaves' extract of $C$. carandas by El-Desoky et al. [70], and the authors have also reported their anti-inflammatory and antioxidant activities (Table 3). The volatile phenolic compound (2-hydroxyacetophenone (13)), having antibacterial potential (Table 4), was reported for the first time from C. lanceolate (syn. C. spinarum) by Hettiarachchi et al. [39] (Table 4). Three flavonoids (kaempferol-3-Orobinobioside (42), kaempferol-3-O- $\alpha$-L-rhamnopyranosyl (1-6)(4"- $p$-coumaroyl) $\beta$-D- galactopyranoside $7-O-\alpha$-L-rhamnopyrano-side (45), and variabiloside $E(46))$, one phenolic acid (p-coumaric acid (43)) along with two phenolic glycosides (salicin (44) and 3,4-dimethylphenol $\beta$-gentiobioside (47)) were isolated from leaves of $C$. macrocarpa, and all compounds have been screened for cytotoxicity activity [71] (Table 5). Begum et al. [49] extracted 4-hydroxybenzoic acid (a phenolic acid) from the leaves of $C$. carandas. Two flavones (epigenin and luteolin) have been extracted by Parveen et al. [72] from the aerial parts of C. opaca (syn. C. spinarum). In the year 2021, Liu et al. [73] isolated a total of ten phenolic compounds, such as $(6 R, 7 S, 8 S)$ 7a-[( $\beta$-D-glucopyranosyl)-oxy]-1-methoxyisolariciresinol (37), (+)-isolariciresinol3a-O- $\beta$-Dglucopyranoside (38), (+)-lyoniresinol3 $\alpha$-O- $\beta$-D-glucopyranoside (40), (-)-lyoniresinol $3 \alpha-O$ $\beta$-D-glucopyranoside (39), acetophenone-2-O- $\beta$ xylopyranosyl-( $(\rightarrow 6)-O$ - $\beta$-glucopyranoside (41), erythro-1-(3- methoxy-4-hydroxy-phenyl)-propan-1,2-diol (32), threo-1-(3-methoxy-4hydroxy-phenyl)-propan-1,2-diol (34), 3-carboxymethyl-benzoic acid (33), protocatechuic acid (36), and and vanillic acid (35), from the root bark of C. spinarum. The authors have also screened these compounds for their antioxidant activity [73] (Table 3).

Table 3. Antioxidant potential of Carissa species.

\begin{tabular}{|c|c|c|c|c|c|}
\hline Carissa Species & $\mathrm{IC}_{50} / \mathrm{EC}_{50} / \mathrm{SC}_{50}$ & Plant Parts & Solvent Used/Compounds & $\begin{array}{l}\text { Major } \\
\text { Findings }\end{array}$ & Reference \\
\hline \multicolumn{6}{|c|}{ DPPH Assay } \\
\hline \multirow{4}{*}{ C. spinarum (syn. C. opaca) } & \multirow{4}{*}{$\begin{array}{c}\mathrm{EC}_{50} \\
(\mu \mathrm{g} / \mathrm{mL})\end{array}$} & Leaves & \multirow{3}{*}{$\begin{array}{l}\text { Methanol, butanol, chloroform, } \\
\text { hexane, ethyl acetate, aqueous }\end{array}$} & $38-500,000$ & [74] \\
\hline & & Fruits & & $59-250$ & [75] \\
\hline & & Roots & & $290-5530$ & [17] \\
\hline & & Leaves & Methanol & 499.95 & [76] \\
\hline \multirow{2}{*}{ C. spinarum (syn. C. edulis) } & $\begin{array}{c}\mathrm{IC}_{50} \\
(\mu \mathrm{g} / \mathrm{mL})\end{array}$ & Fruits & $\begin{array}{l}\text { Petroleum ether, ethyl acetate, } \\
\text { chloroform, ethanol, water }\end{array}$ & $169-488$ & [77] \\
\hline & $\mathrm{SC}_{50}(\mu \mathrm{g})$ & Stem & Chloroform & 47.04 & {$[78]$} \\
\hline C. spinarum & $\begin{array}{c}\mathrm{IC}_{50} \\
(\mu \mathrm{g} / \mathrm{mL})\end{array}$ & Root bark & $\begin{array}{l}\text { Chloroform, petroleum ether, } \\
\text { dichloromethane, ethyl acetate, } \\
n \text {-butanol }\end{array}$ & $31.8-500$ & [74] \\
\hline
\end{tabular}


Table 3. Cont.

\begin{tabular}{|c|c|c|c|c|c|}
\hline Carissa Species & $\mathrm{IC}_{50} / \mathrm{EC}_{50} / \mathrm{SC}_{50}$ & Plant Parts & Solvent Used/Compounds & $\begin{array}{l}\text { Major } \\
\text { Findings }\end{array}$ & Reference \\
\hline \multirow{7}{*}{ C. carandas } & \multirow{2}{*}{$\begin{array}{c}\mathrm{EC}_{50} \\
(\mu \mathrm{g} / \mathrm{mL})\end{array}$} & \multirow{2}{*}{ Leaves } & \multirow{2}{*}{ Methanol } & 630.4 & {$[79]$} \\
\hline & & & & 10.5 & [80] \\
\hline & \multirow{5}{*}{$\begin{array}{c}\mathrm{IC}_{50} \\
(\mu \mathrm{g} / \mathrm{mL})\end{array}$} & & \multirow{2}{*}{ Ethanol } & 1.292 & [44] \\
\hline & & Leaves & & 1.47 & [76] \\
\hline & & & \multirow{2}{*}{ Methanol } & 73.1 & [81] \\
\hline & & Fruits & & 27.4 & [52] \\
\hline & & Leaves and fruits & Chloroform and ethanol & $195.8-259.5$ & [82] \\
\hline \multirow{3}{*}{ C. macrocarpa } & \multirow{3}{*}{$\begin{array}{c}\mathrm{EC}_{50} \\
(\mu \mathrm{g} / \mathrm{mL})\end{array}$} & Fruit & \multirow{3}{*}{ Hydroethanolic } & 9900 & [46] \\
\hline & & Leaves & & 26 & \multirow{2}{*}{ [83] } \\
\hline & & Stem & & 281 & \\
\hline \multirow{2}{*}{ C. bispinosa } & \multirow{2}{*}{$\mathrm{IC}_{50}$} & \multirow{2}{*}{ Fruits } & Fraction $1\left(\mathrm{R}_{\mathrm{f}=}=0.11\right)$ & 127.50 & \multirow{2}{*}{ [9] } \\
\hline & & & Fraction $2\left(R_{f}=0.38\right)$ & 183.38 & \\
\hline & & ABTS assay & & & \\
\hline \multirow{2}{*}{ C. spinarum (syn. C. opaca) } & \multirow{3}{*}{$\begin{array}{c}\mathrm{EC}_{50} \\
(\mu \mathrm{g} / \mathrm{mL})\end{array}$} & Leaves & \multirow{2}{*}{$\begin{array}{l}\text { Methanol, butanol, chloroform, } \\
\text { hexane, ethyl acetate, aqueous }\end{array}$} & $70-187$ & [84] \\
\hline & & Fruits & & $80-500$ & {$[75]$} \\
\hline C. carandas & & Leaves & Methanol & 1.75 & {$[80]$} \\
\hline \multicolumn{6}{|c|}{ Phsophomolybdate or total antioxidant assay } \\
\hline \multirow{2}{*}{ C. spinarum (syn. C. opaca) } & \multirow{2}{*}{$\begin{array}{c}\mathrm{EC}_{50} \\
(\mu \mathrm{g} / \mathrm{mL})\end{array}$} & Leaves & \multirow{2}{*}{$\begin{array}{l}\text { Methanol, butanol, chloroform, } \\
\text { hexane, ethyl acetate, aqueous }\end{array}$} & $30-250$ & [74] \\
\hline & & Fruits & & $86-500$ & {$[75]$} \\
\hline & & Thiobarbituric ass & & & \\
\hline C. spinarum (syn. C. edulis) & $\begin{array}{c}\mathrm{IC}_{50} \\
(\mu \mathrm{g} / \mathrm{mL})\end{array}$ & Fruits & $\begin{array}{l}\text { Petroleum ether, ethyl acetate, } \\
\text { chloroform, ethanol, water }\end{array}$ & $114-289$ & [77] \\
\hline & & Fruit & & 1230 & {$[46]$} \\
\hline C. macrocarpa & $\mathrm{EC}_{50}$ & Leaves & Hydroethanolic & 15.4 & {$[83]$} \\
\hline & & Stem & & 12.1 & {$[00]$} \\
\hline & & $\beta$-carotene assay & & & \\
\hline$C$ sninarum (sun $C$ onaca) & $\mathrm{EC}_{50}$ & Leaves & Methanol, butanol, chloroform & $145-250$ & [74] \\
\hline 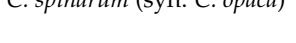 & $(\mu \mathrm{g} / \mathrm{mL})$ & Fruits & hexane, ethyl acetate, aqueous & $233.8-8000$ & [75] \\
\hline & & Fruit & & 880 & {$[46]$} \\
\hline C. macrocarpa & $\begin{array}{c}\mathrm{EC}_{50} \\
(\mu \mathrm{g} / \mathrm{mL})\end{array}$ & Leaves & Hydroethanolic & 300 & [83] \\
\hline & & Stem & & 270 & {$[03]$} \\
\hline & & en peroxide scaveng & activity & & \\
\hline C sninarum (syn $C$ onaca) & $\mathrm{EC}_{50}$ & Leaves & Methanol, butanol, chloroform & $19-250$ & {$[74]$} \\
\hline C. spentarmin (sуп. (. ориса) & $(\mu \mathrm{g} / \mathrm{mL})$ & Fruits & hexane, ethyl acetate, aqueous & $47.2-250$ & [75] \\
\hline C. spinarum (syn. C. edulis) & $\begin{array}{c}\mathrm{IC}_{50} \\
(\mu \mathrm{g} / \mathrm{mL})\end{array}$ & Fruits & $\begin{array}{l}\text { Petroleum ether, ethyl acetate, } \\
\text { chloroform, ethanol, water }\end{array}$ & $138-503$ & [74] \\
\hline & & & Ethanol & 2.038 & \\
\hline C. carandas & $\begin{array}{c}\mathrm{IC}_{50} \\
(\mu \mathrm{g} / \mathrm{mL})\end{array}$ & Leaves & $n$-Hexane & 1.802 & [44] \\
\hline & & & Methanol & 84.0 & [81] \\
\hline & & ide radical scaveng & activity & & \\
\hline & & Leaves & & 93-206 & [74] \\
\hline C. spinarum (syn. C. opaca) & $(\mu \mathrm{g} / \mathrm{mL})$ & Fruits & hexane, ethyl acetate, aqueous & $33.43-250$ & {$[75]$} \\
\hline & & kyl radical scavengi & ctivity & & \\
\hline & & Leaves & Methanol, butanol, chloroform & $18-22$ & [74] \\
\hline C. spinarum (syn. C. opaca) & $\begin{array}{c}\mathrm{EC}_{50} \\
(\mu \mathrm{g} / \mathrm{mL})\end{array}$ & Fruits & hexane, ethyl acetate, aqueous & $36.24-250$ & {$[75]$} \\
\hline C. carandas & & Leaves & Methanol (E) & 606.9 & [79] \\
\hline & & Chelating powe & & & \\
\hline & $\mathrm{EC}_{50}$ & Leaves & & $16-137$ & [74] \\
\hline C. spinarum (syn. C. opaca) & $(\mu$ & Fruits & hexane, ethyl acetate, aqueous & $25.1-46.3$ & [75] \\
\hline
\end{tabular}


Table 3. Cont.

\begin{tabular}{|c|c|c|c|c|c|}
\hline Carissa Species & $\mathrm{IC}_{50} / \mathrm{EC}_{50} / \mathrm{SC}_{50}$ & Plant Parts & Solvent Used/Compounds & $\begin{array}{c}\text { Major } \\
\text { Findings }\end{array}$ & Reference \\
\hline \multicolumn{6}{|c|}{ Reducing power assay } \\
\hline C. spinarum (syn. C. edulis) & $\begin{array}{l}\mathrm{IC}_{50} \\
(\mu \mathrm{g})\end{array}$ & Fruits & $\begin{array}{l}\text { Petroleum ether, ethyl acetate, } \\
\text { chloroform, ethanol, water }\end{array}$ & $109-240$ & [77] \\
\hline \multirow{2}{*}{ C. carandas } & \multirow{2}{*}{$\begin{array}{c}\mathrm{EC}_{50} \\
(\mu \mathrm{g} / \mathrm{mL})\end{array}$} & Fruits & Fruit wine & 110300 & [85] \\
\hline & & Leaves & Methanol & 62.09 & [79] \\
\hline \multirow{3}{*}{ C. macrocarpa } & \multirow{3}{*}{$\begin{array}{c}\mathrm{EC}_{50} \\
(\mu \mathrm{g} / \mathrm{mL})\end{array}$} & Fruit & \multirow{3}{*}{ Hydroethanolic } & 1590 & [46] \\
\hline & & Leaves & & 36 & \multirow{2}{*}{ [83] } \\
\hline & & Stem & & 33 & \\
\hline \multicolumn{6}{|c|}{ Isolated compounds of genus Carissa } \\
\hline \multicolumn{6}{|c|}{ DPPH assay } \\
\hline \multirow{10}{*}{ C. spinarum } & \multirow{8}{*}{$\begin{array}{l}\mathrm{IC}_{50} \\
(\mu \mathrm{M})\end{array}$} & \multirow{8}{*}{ Stem } & Olivil & 18.1 & \multirow{8}{*}{ [23] } \\
\hline & & & Carinol & 20.2 & \\
\hline & & & Secoisolariciresinol & 26.2 & \\
\hline & & & Carissanol & 33.4 & \\
\hline & & & Cycloolivil & 33.2 & \\
\hline & & & Nortrachelogenin & 35.8 & \\
\hline & & & Pinoresinol & 43.4 & \\
\hline & & & (+)-8-Hydroxypinoresinol & 69.5 & \\
\hline & \multirow{2}{*}{$\begin{array}{l}\mathrm{IC}_{50} \\
(\mu \mathrm{M})\end{array}$} & \multirow{2}{*}{ Root bark } & $\begin{array}{l}\text { Isolariciresinol3a-O- } \beta \text {-D- } \\
\text { glucopyranoside }\end{array}$ & 16.5 & \multirow{2}{*}{ [73] } \\
\hline & & & Protocatechuic acid & 45.7 & \\
\hline \multirow{2}{*}{ C. spinarum } & \multirow{2}{*}{$\begin{array}{l}\mathrm{SC}_{50} \\
(\mu \mathrm{M})\end{array}$} & \multirow{2}{*}{ Stem } & Carissanol & 37.12 & \multirow{2}{*}{ [78] } \\
\hline & & & Carinol & 47.87 & \\
\hline \multirow{6}{*}{ C. carandas } & \multirow{5}{*}{$\begin{array}{l}\mathrm{IC}_{50} \\
(\mu \mathrm{M})\end{array}$} & \multirow{5}{*}{ Stem } & Carandoside & 116.5 & \multirow{5}{*}{ [56] } \\
\hline & & & $\begin{array}{c}(6 S, 7 R, 8 R)-7 \mathrm{a}-[(\beta- \\
\text { glucopyranosyl)oxy]lyoniresinol }\end{array}$ & 21.5 & \\
\hline & & & $\begin{array}{c}(6 R, 7 S, 8 S)-7 \mathrm{a}-[(\beta- \\
\text { glucopyranosyl)oxy]lyoniresinol }\end{array}$ & 43 & \\
\hline & & & Carissanol & 12.7 & \\
\hline & & & Nortrachelogenin & 30.2 & \\
\hline & $\begin{array}{l}\mathrm{EC}_{50} \\
(\mu \mathrm{M})\end{array}$ & Leaves & Naringin & 11.2 & [70] \\
\hline \multicolumn{6}{|c|}{ Superoxide radical scavenging activity } \\
\hline C. carandas & $\begin{array}{l}\mathrm{EC}_{50} \\
(\mu \mathrm{M})\end{array}$ & Leaves & Naringin & 0.08 & [70] \\
\hline
\end{tabular}

$\left(\mathrm{EC}_{50}\right)$ Half maximal effective concentration. ( $\left.\mathrm{IC}_{50}\right)$ Inhibitory concentration required for $50 \%$ inhibition. $\left(\mathrm{SC}_{50}\right)$ Scavenging concentration required for $50 \%$ scavenging.

Table 4. Antimicrobial potential of Carissa species.

\begin{tabular}{cccccc}
\hline Carissa Species & Plant Part Used & Extract/Compound & Microorganisms & MIC (mg/mL) & References \\
\hline $\begin{array}{c}\text { C. spinarum } \\
\text { (syn. C. opaca) }\end{array}$ & Roots & Ethyl acetate & $\begin{array}{c}\text { Pseudomonas aeruginosa, } \\
\text { Bacillus subtilis }\end{array}$ & 0.007-0.008 & {$[35]$} \\
\hline C. spinarum & Leaves and roots & Methanol and ethanol & $\begin{array}{c}\text { Escherichia coli, } \\
\text { Staphylococcus aureus }\end{array}$ & 0.312-2.5 \\
\hline $\begin{array}{c}\text { C. spinarum }(\text { syn. } \\
\text { C. lanceolata) }\end{array}$ & Roots & $\begin{array}{c}\text { Root bark methanol, } \\
\text { root bark } \\
\text { dichloromethane, } \\
\text { root wood methanol, root } \\
\text { wood, } \\
\text { dichloromethane }\end{array}$ & $\begin{array}{c}\text { Bacillus subtilis, } \\
\text { Escherichia coli }\end{array}$ & $2.5-20$ \\
\hline
\end{tabular}


Table 4. Cont.

\begin{tabular}{|c|c|c|c|c|c|}
\hline Carissa Species & Plant Part Used & Extract/Compound & Microorganisms & $\mathrm{MIC}(\mathrm{mg} / \mathrm{mL})$ & References \\
\hline \multirow[t]{2}{*}{ C. carandas } & Fruits & Dichloromethane & $\begin{array}{l}\text { Staphylococcus aureus, } \\
\text { Escherichia coli, } \\
\text { Klebsiella pneumonia, } \\
\text { Enterococcus faecalis }\end{array}$ & $0.31-5$ & [87] \\
\hline & $\begin{array}{l}\text { Leaves, stems, } \\
\text { and roots }\end{array}$ & $\begin{array}{l}\text { Petroleum ether, water, } \\
\text { and methanol }\end{array}$ & $\begin{array}{c}\text { Bacillus subtilis, } \\
\text { Agrobacterium tumifaciens, } \\
\text { Pseudomonas aeruginosa }\end{array}$ & $0.078-1.25$ & [88] \\
\hline \multirow{3}{*}{ C. macrocarpa } & Fruits & Hydroethanolic & $\begin{array}{c}\text { Escherichia coli, } \\
\text { Pseudomonas aeruginosa, } \\
\text { Enterococcus faecalis }\end{array}$ & $10-20$ & [46] \\
\hline & $\begin{array}{l}\text { Leaves, stems, } \\
\text { and flower }\end{array}$ & Hydroethanolic & $\begin{array}{c}\text { Escherichia coli, } \\
\text { Enterococcus faecalis, Listeria } \\
\text { monocytogenes }\end{array}$ & $0.62-20$ & [84] \\
\hline & $\begin{array}{l}\text { Fruits, stems, } \\
\text { and flowers }\end{array}$ & Essential oil & $\begin{array}{l}\text { Salmonella enterica, } \\
\text { Staphylococcus aureus, } \\
\text { Bacillus subtilis }\end{array}$ & $0.46-7.5$ & [71] \\
\hline $\begin{array}{l}\text { C. macrocarpa (Syn. } \\
\text { C. grandiflora) }\end{array}$ & $\begin{array}{l}\text { Stems, roots, } \\
\text { and leaves }\end{array}$ & $\begin{array}{l}\text { Methanol, } n \text {-butanol, } \\
\text { ethyl acetate, chloroform, } \\
n \text {-hexane }\end{array}$ & $\begin{array}{c}\text { Staphylococcus aureus, } \\
\text { Escherichia coli, } \\
\text { Staphylococcus epidermidis }\end{array}$ & $0.24-2.69$ & [89] \\
\hline \multicolumn{6}{|c|}{ Antifungal activity of Carissa species } \\
\hline \multirow[t]{2}{*}{ C. spinarum (syn. C. opaca) } & Roots & $\begin{array}{l}\text { Ethyl acetate, } \\
\text { acetone }\end{array}$ & $\begin{array}{l}\text { Candida albicans, } \\
\text { Alternaria solani, } \\
\text { Aspergillus flavus }\end{array}$ & $0.05-0.1$ & [34] \\
\hline & Roots & Ethyl acetate & Candida albicans & 0.007 & [7] \\
\hline C. macrocarpa & Fruits & Essential oil & Candida albicans & 0.46 & [15] \\
\hline \multicolumn{6}{|c|}{ Antibacterial activity of some isolated compounds from Carissa species } \\
\hline \multirow{3}{*}{$\begin{array}{l}\text { C. spinarum (syn. } \\
\text { C. lanceolata) }\end{array}$} & \multirow{3}{*}{ Wood } & Dehydrocarissone (15) & \multirow{3}{*}{$\begin{array}{c}\text { Staphylococcus aureus, } \\
\text { Escherichia coli, } \\
\text { Pseudomonas aeruginosa }\end{array}$} & $0.5-2$ & \multirow{3}{*}{ [1] } \\
\hline & & Carindone (16) & & $0.5-1$ & \\
\hline & & Carissone (3) & & $0.1-2$ & \\
\hline \multirow{3}{*}{$\begin{array}{l}\text { C. spinarum (syn. } \\
\text { C. lanceolata) }\end{array}$} & Roots & $\begin{array}{c}2- \\
\text { Hydroxyacetophenone (13) }\end{array}$ & \multirow{3}{*}{$\begin{array}{c}\text { Bacillus subtilis, } \\
\text { Staphylococcus aureus, } \\
\text { Escherichia coli, } \\
\text { Pseudomonas aeruginosa }\end{array}$} & 1.25 & \multirow{3}{*}{ [39] } \\
\hline & & Carinol (6) & & 1.25 & \\
\hline & & Carissone (3) & & $5-10$ & \\
\hline C. macrocarpa & Fruits & $\begin{array}{c}\text { 3ß-Hydroxyolean-11-en- } \\
28,13 \\
\beta \text {-olide (14) }\end{array}$ & $\begin{array}{c}\text { Escherichia coli, } \\
\text { Enterococcus faecium, } \\
\text { Staphylococcus } \\
\text { saprophyticus, } \\
\text { Klebsiella } \\
\text { pneumonia,Pseudomonas } \\
\text { aeruginosa, } \\
\text { Staphylococcus aureus }\end{array}$ & $0.06-0.12$ & [8] \\
\hline
\end{tabular}

Table 5. In vitro cytotoxic activity of Carissa species.

\begin{tabular}{|c|c|c|c|c|c|c|}
\hline Species & $\begin{array}{l}\text { Part } \\
\text { Used }\end{array}$ & $\begin{array}{l}\text { Solvent } \\
\text { Used }\end{array}$ & Isolated Compounds & Cell Lines & $\begin{array}{c}\text { Major } \\
\text { Findings }\end{array}$ & References \\
\hline \multicolumn{7}{|c|}{ In vitrocytotoxic activity of Carissa species $\left(\mathrm{IC}_{50}\right.$ in $\left.\mu \mathrm{g} / \mathrm{mL}\right)$} \\
\hline \multirow[t]{2}{*}{ C. carandas } & Fruits & Methanol & - & $\begin{array}{c}\text { Cervical cell line (Hela), } \\
\text { Breast cancer (MCF7), } \\
\text { Hepatocellular carcinoma } \\
\text { (HeptG2), } \\
\text { Bone sarcoma (MG-63) }\end{array}$ & $56.72-86.91$ & [90] \\
\hline & Leaves & Methanol & & $\begin{array}{c}\text { Kidney carcinoma (A-498), } \\
\text { Prostate carcinoma (PC-3), } \\
\text { Embryonic lung tissue (L-132) }\end{array}$ & $55.56-240$ & [91] \\
\hline
\end{tabular}


Table 5. Cont.

\begin{tabular}{|c|c|c|c|c|c|c|}
\hline Species & $\begin{array}{l}\text { Part } \\
\text { Used }\end{array}$ & $\begin{array}{l}\text { Solvent } \\
\text { Used }\end{array}$ & Isolated Compounds & Cell Lines & $\begin{array}{c}\text { Major } \\
\text { Findings }\end{array}$ & References \\
\hline \multirow{4}{*}{ C. spinarum } & NM & $\begin{array}{l}\quad n- \\
\text { Hexane, } \\
\text { chloroform, } \\
\text { methanol }\end{array}$ & & Melanoma cells (A375) & $40-100$ & [92] \\
\hline & \multirow{3}{*}{ Stems } & \multirow{3}{*}{ NM } & Carissanol (5) & \multirow{3}{*}{$\begin{array}{l}\text { Normal human (WI-38), } \\
\text { Human lung (A549), } \\
\text { Human breast (MCF7) }\end{array}$} & $6-17$ & \multirow{3}{*}{ [23] } \\
\hline & & & Carinol (6) & & $<1$ & \\
\hline & & & Nortrachelogenin (23) & & $29-100$ & \\
\hline $\begin{array}{l}\text { C. spinarum (syn. } \\
\text { C. congesta) }\end{array}$ & Stems & $\begin{array}{c}n- \\
\text { Butanol } \\
\end{array}$ & & Leukaemia cells (HL-60) & 34.58 & [93] \\
\hline $\begin{array}{l}\text { C. spinarum(Syn. } \\
\text { C. edulis) }\end{array}$ & $\begin{array}{l}\text { Fresh } \\
\text { fruits }\end{array}$ & \multicolumn{2}{|l|}{ Ethanol } & Lung cancer (A549) & 405 & [77] \\
\hline \multirow[b]{6}{*}{$\begin{array}{l}\text { C. spinarum (syn. } \\
\text { C. edulis) }\end{array}$} & \multicolumn{5}{|c|}{ In vitrocytotoxic activity of Carissa species $\left(\mathrm{IC}_{50}\right.$ in $\left.\mu \mathrm{M}\right)$} & 171 \\
\hline & \multirow[b]{5}{*}{ Root bark } & \multirow[b]{5}{*}{ Methanol } & Carissaedulosides A (24) & \multirow[b]{5}{*}{$\begin{array}{c}\text { Human leukaemia (HL-60), } \\
\text { Lung cancer (A549), } \\
\text { Breast cancer (MCF-7), } \\
\text { Colon cancer (SW480) }\end{array}$} & $10-20$ & \multirow[b]{5}{*}{ [94] } \\
\hline & & & Carissaedulosides D (25) & & $10-22$ & \\
\hline & & & Carissaedulosides J (26) & & $3.8-17$ & \\
\hline & & & Sarhamnoloside (22) & & $0.02-0.13$ & \\
\hline & & & $\begin{array}{c}\text { [(1S,2S,3S)-1,2,3,4- } \\
\text { tetrahydro-3,7-dihydroxy-1- } \\
\text { (4-hydroxy-3 } \\
\text { methoxyphenyl)-3 } \\
\text { (hydroxymethyl)-6- } \\
\text { methoxy-2-naphthalen-yl] } \\
\text { methyl } \\
\beta \text {-D-glucopyranoside (51) }\end{array}$ & & $5.6-19$ & \\
\hline \multirow[b]{2}{*}{ C. carandas } & Leaves & NM & Carandinol (17) & $\begin{array}{c}\text { HeLa (Cervical cancer), } \\
\text { PC-3 (Prostate cancer), } \\
\text { 3T3 (Normal mouse fibroblast) }\end{array}$ & $6.87-12.60$ & [41] \\
\hline & $\begin{array}{l}\text { Fresh } \\
\text { leaves }\end{array}$ & NM & $\begin{array}{l}\beta \text {-sitosterol-3-O- } \beta \text {-D- } \\
\text { glucopyranoside } \\
\quad(55)\end{array}$ & $\begin{array}{c}\text { Small cell lung carcinoma } \\
\text { (NCI-H460), } \\
\text { Oral squamous cell carcinoma } \\
\text { (Cal-27), } \\
\text { Normal mouse fibroblast }\end{array}$ & $18.6-63.3$ & [95] \\
\hline \multirow{3}{*}{ C. macrocarpa } & \multirow{3}{*}{ Leaves } & \multirow{3}{*}{ Methanol } & $\begin{array}{c}\text { Kaempferol } \\
\text { 3-O-robinobioside (42) }\end{array}$ & \multirow{3}{*}{$\begin{array}{l}\text { Human lung cancer } \\
\text { (A549) }\end{array}$} & 93.6 & \multirow{3}{*}{ [71] } \\
\hline & & & $\begin{array}{c}\text { Kaempferol-3-O- } \alpha \text {-L- } \\
\text { rhamnopyranosyl } \\
(1-6)\left(4^{\prime \prime}-p \text {-coumaro-yl) } \beta \text {-D- }\right. \\
\text { galacto-pyranoside7-O- } \alpha \text {-L- } \\
\text { rhamno-pyranoside }(45)\end{array}$ & & 100.4 & \\
\hline & & & Variabiloside E (46) & & 84.3 & \\
\hline \multicolumn{7}{|c|}{ In vitrocytotoxic activity of Carissa species (Inhibition in \%) } \\
\hline $\begin{array}{l}\text { C. spinarum (syn. } \\
\text { C. opaca) }\end{array}$ & Leaves & $\begin{array}{l}\text { Chloroform } \\
\text { ethyl ac- } \\
\text { etate, } \\
\text { methanol }\end{array}$ & - & Breast cancer (MCF-7) & $78-99$ & [60] \\
\hline \multirow[b]{2}{*}{ C. carandas } & \multirow[b]{2}{*}{ Fruits } & \multicolumn{2}{|c|}{ Aqueous ethanol } & HeLa cancer cell & 67.87 & [87] \\
\hline & & Methanol & - & $\begin{array}{l}\text { Breast (MCF-7), } \\
\text { Colon (HCT-116), } \\
\text { Lung (A-549), } \\
\text { Ovarian (OVCAR-5), } \\
\text { Prostate (PC-3) }\end{array}$ & $63-100$ & [96] \\
\hline
\end{tabular}


Table 5. Cont.

\begin{tabular}{|c|c|c|c|c|c|c|}
\hline Species & $\begin{array}{l}\text { Part } \\
\text { Used }\end{array}$ & $\begin{array}{l}\text { Solvent } \\
\text { Used }\end{array}$ & Isolated Compounds & Cell Lines & $\begin{array}{c}\text { Major } \\
\text { Findings }\end{array}$ & References \\
\hline \multicolumn{7}{|c|}{ In vitrocytotoxic activity of Carissa species $\left(\mathrm{EC}_{50}\right.$ in $\left.\mu \mathrm{g} / \mathrm{mL}\right)$} \\
\hline \multirow{2}{*}{ C. carandas } & Leaves & Chloroform & - & $\begin{array}{l}\text { Human ovarian carcinoma } \\
\text { (Cavo 3) }\end{array}$ & 7.702 & \multirow{2}{*}{ [97] } \\
\hline & $\begin{array}{l}\text { unripe } \\
\text { fruits }\end{array}$ & $\begin{array}{c}n- \\
\text { Hexane }\end{array}$ & - & Lung cancer (NCL) & 2.492 & \\
\hline \multicolumn{7}{|c|}{ In vitrocytotoxic activity of Carissa species $\left(\mathrm{GI}_{50}\right.$ in $\mu \mathrm{g} / \mathrm{mL}$ ) } \\
\hline $\begin{array}{l}\text { C. spinarum (syn. } \\
\text { C. congesta) }\end{array}$ & Roots & $\begin{array}{l}\text { Petroleum } \\
\text { ether }\end{array}$ & & Breast cancer (MCF7) & 18.1 & [98] \\
\hline \multirow[t]{2}{*}{ C. macrocarpa } & $\begin{array}{c}\text { Leaves, } \\
\text { stems, } \\
\text { and flow- } \\
\text { ers }\end{array}$ & Hydroethanolic & & \multirow{2}{*}{$\begin{array}{c}\text { Breast carcinoma (MCF-7), } \\
\text { Cervical carcinoma(HeLa), } \\
\text { Non-small cell lungcarcinoma } \\
\text { (NCI-H460), } \\
\text { Hepatocellular carcinoma } \\
\text { (HepG2) }\end{array}$} & $52-400$ & [83] \\
\hline & Fruits & - & & & $57-400$ & [46] \\
\hline
\end{tabular}

(-) Not reported.

\subsection{Lignans}

A total of thirty lignans have been reported from different species of Carissa. Rao et al. [78] isolated a total of seven lignans (coniferaldehyde, pinoresinol (31), nortrachelogenin (23), carissanol (5), secoisolariciresinol (29), carinol (6), and olivil (18)) from the chloroform extract of $C$. spinarum stem, and out of these compounds, only carissanol (5) and carinol (6) showed antioxidant potential. Similarly, Wangteeraprasert and Likhitwitayawuid [56] eluted three lignans ((6R,7S,8S)-7a-[(b-glucopyranosyl)oxy]lyoniresinol, $(6 S, 7 R, 8 R)-7 \mathrm{a}-[(\beta-$ glucopyranosyl)oxy] lyoni-resinol (49), carissanol (5), and nortrachelogenin (23)) from the stem of $C$. carandas. These isolated compounds were also screened by the authors for their antioxidant activity [56] (Table 3). From the C. lanceolate (syn. C. spinarum) root, carinol (6) was reported by Hettiarachchi et al. [39]. Additionally, a total of eleven lignans (carinol (6), olivil (18), cycloolivil (28), 8-hydroxypinoresinol (30), secoisolariciresinol (29), digitoxigenin-3-O$\beta$-D-digitalopyranoside, nortrachelogenin (23), evomonoside, scopoletin (8), (+)-pinoresinol (31), and carissanol (5)) have been extracted from the methanol extract of $C$. spinarum stem, followed by the analysis of their free radical scavenging (DPPH assay) and cytotoxic activities [23] (Tables 3 and 5). Other lignans, nortrachelogenin (23), carinol (6), olivil (18), and carissanol (5), were isolated from the methanolic root extract of C. edulis (syn. C. spinarum) by Achenbach et al. [99].

Kaunda et al. [94] isolated three furofuran lignans (carissaeduloside $\mathrm{H}$, carissaeduloside I, and carissaeduloside J (26)) together with 12 analogues (sarhamnoloside (22), [(1S,2S,3S)-1,2,3,4tetrahydro-3,7-dihydroxy-1-(4-hydroxy-3-methoxyphenyl)-3-(hydroxymethyl)-6-methoxy-2naphthalen-yl] methyl $\beta$-D-glucopyranoside (51), (-)-lyoniresinol 9-O- $\beta$-D-glucopyranoside, $(-)$-lyoniresinol 9'-O-D-glucopyranoside, guaiacylglycerol, (+)-1-acetoxypinoresinol-4 $4^{\prime}-\beta$-Dglucoside4"-O-methyl ether, (+)-1-acetoxypinoresinol4'- $\beta$-D-glucoside, 1-(1-hydroxyethyl)-2-(6(1-hydroxyethyl) phenoxy)benzene, scopoletin (8), khaephuoside A, 3,4-dimethoxyphenyl 2-O$\beta$-D-apiofuranosyl-(1 $\rightarrow 2)-\beta$-D-glucopyranoside, and markhamioside $F)$ from the root bark of C. edulis (syn. C. spinarum). Out of these compounds, sarhamnoloside (22) and [(1S,2S,3S)-1,2,3,4tetrahydro-3,7-dihydroxy-1-(4-hydroxy-3-methoxyphenyl)-3-(hydroxyl-methyl)-6-methoxy-2naphthalen-yl] methyl $\beta$-D-glucopyranoside (51) exhibited anticancer activity against lung and breast cancer cell lines [94] (Table 5).

\subsection{Terpenoids}

A total of 41 terpenes and terpenoids were reported from the Carissa species. In 1985, the compound carissol (12), an epimer of $\alpha$-amyrin (21), was isolated from C. carandas [100]. The methanolic extract from the root of C. edulis (syn. C. spinarum) was characterised with seven sesquiterpenes (eudesmane-type and a novel germacrane derivate), 
e.g., carissone, cryptomeridiol, $\beta$-eudesmol, germacrenone, $6 \beta$-carissanol, $6 \alpha$-carissanol, and $\alpha$-carissanol [101]. Whereas C. carandas fruits were rich in two triterpenes (carissic acid (11) and carissic acid methyl ester) [102]. From the dichloromethane extract of the wood of C. lanceolata (syn. C. spinarum), three terpenoids (carissone (3), dehydrocarissone (15), and carindone (16)) have been isolated by Lindsay et al. [1] and also reported for their antibacterial activity (Table 4). Four pentacyclic triterpenoids (oleanolic acid (54), ursolic acid (2), carissin, and 3- $\beta$-hydroxy-27- $p$-E-coumaroyloxyurs-12-en-28-oic acid) were isolated for the first time by Siddiqui et al. [102] from the leaves of C. carandas. Similarly, Rao et al. [78] isolated germacrane sesquiterpenes (carenone (10), $3^{\prime}$-( $4^{\prime \prime}$-methoxyphenyl)$3^{\prime}$-oxo-propionylhexadecanoate, and germacrenone) from the $C$. spinarum stem.

From the petroleum ether root extract of $C$. carandas, three triterpenoids (lupa-12,20(29)dien-3b,28-diol, urs-12-ene-3 $\beta, 22 \beta$-diol, and ursolic acid (2)) were isolated [103]. One new compound, carandoside (48), was extracted and analysed for its antioxidant activity from the stem of C. carandas [56] (Table 4). The compounds lupeol (1), $16 \beta$-hydroxy betulinic acid, and $\alpha$-amyrin (21) were reported for the first time from the roots of $C$. carandas by Hegde and Joshi [104,105]. Tolo et al. [84] isolated four terpenoids (lupeol (1), oleuropein (20), carissol (12), and $\beta$-amyrin) from the root bark of $C$. edulis (syn. C. spinarum) and screened these compounds for antiviral activity [106]. Likewise, Moodley et al. [8] isolated four pentacyclic oleanane triterpenes ( $\beta$-amyrin (52), methyl oleanolate (53), oleanolic acid (54), and $3 \beta$ hydroxyolean-11-en-28,13 $\beta$-olide (14)) from the fruits of $C$. macrocarpa, while the ursane-type triterpene, ursolic acid (2), was isolated from the leaves of $C$. macrocarpa and also reported by the authors for antibacterial activity (Table 4).

Two triterpenoids, namely $\beta$-amyrin and lupeol (1), have been isolated from C. edulis (syn. C. spinarum) [68]. Carissone (3) (a terpenoid) was isolated by Joshi and Boyce [107], Hettiarachchi et al. [39], and Wangteeraprasert et al. [23] from the roots of C. congesta (syn. C. spinarum), the roots of C. lanceolate, and the stem of C. spinarum, respectively. The compound has also been screened for free radical scavenging activity (DPPH) and cytotoxic activity [23] (Tables 3 and 5).

Similarly, Karunakar et al. [108] reported triterpenoids such as lupeol (1), ursolic acid (2), 17-hydroxy-11-oxo-nor- $\beta$-amyrone, and urs-12-ene-3 $\beta, 22 \beta$-diol-17-carboxylic acid from a petroleum ether extract of $C$. spinarum roots. Whereas lanostane triterpenoid (lanost-5-en$3 \beta$-ol-21-oic acid (27)) was extracted from the ethanolic extract of the fruit of $C$. carandas by Arif et al. [109], and the authors also reported on its apoptogenic activity. Carandinol (17), a triterpene, was isolated for the first time by Begum et al. [41] from the leaves of C. carandas, along with three known compounds, betulinic acid, oleanolic acid, and ursolic acid (2). Among all these compounds, only carandinol (17) exhibited a cytotoxic effect against cancer cell lines [41] (Table 5). Lupeol (1), carissone (3), and oleanolic acid were isolated from the roots of C. carandas and screened for anti-inflammatory activity [110].

Parveen et al. [111] isolated two new nor-triterpenoids (30-nor-2a,3b-dihydoxyurs-12ene and 30-nor-2a,3b,23-trihydroxyurs-12-ene) from aerial parts of C. opaca (syn. C. spinarum). In the year 2017, Parveen et al. [72] also isolated one more triterpene (ursolic acid (2)) from the aerial parts of C. opaca. Whereas, from the aerial parts of C. opaca (syn. C. spinarum), new nortriterpenoids noropacursane, 6-methoxy-7-hydroxycoumarin, lupeol (1), and 4Ketopinoresinol have been extracted and characterised by Parveen et al. [112]. Additionally, Bano et al. [95] for the first time isolated ursane-type triterpene (sabiracin, para hydroxy benzaldehyde, ursolic acid (2), carissin, and 22a-hydroxyursolic acid) from fresh leaves of C. carandas and reported on their anticancer activity (Table 5).

\subsection{Steroids}

A total of seven steroids were isolated from the Carissa species. Hegde and Joshi [105] reported $\beta$-sitosterol (7) from chloroform extracts of $C$. carandas roots, while $(3 \beta, 5 \alpha)$-Stigma7,16-dien-3-ol, sitosterol glucoside, and $\beta$-sitosterol (7) were isolated from the roots of $C$. congesta (syn. C. spinarum) [107]. The bioactive compounds named stigmasterol glucoside, $\beta$-sitosterol (7), and $\beta$-sitosterol glucoside were isolated by AI-Youssaf and Hassan [69] 
from the aerial parts of $C$. edulis (syn. C. spinarum). From the methanol extract of the aerial parts of $C$. opaca, $\beta$-sitosterol (7) and $\beta$-sitosterol 3-O- $\beta$-D-glucopyranoside (55) have been extracted [72]. Whereas, from the petroleum ether roots extract of $C$. spinarum, stigmasterol and campesterol were isolated by Karunakar et al. [108] using column chromatography. From the leaves of C. carandas, Begum et al. [41] and Bano et al. [95] isolated $\beta$-sitosterol-3$O$ - $\beta$-D-glucopyranoside (55).

\subsection{Coumarins}

Parveen et al. [72] reported one coumarin (6-hydroxy-7-methoxycoumarin) that was isolated from a methanol extract of the aerial parts of C. opaca (syn. C. spinarum). A coumarin-secoirdoid hybrid named carissaeduloside $G$ was isolated from the root bark of C. edulis (syn. C. spinarum) [94].

\subsection{Cardiac Glycosides}

Six glycosides (carissaeduloside A (24), carissaeduloside B, carissaeduloside C, carissaeduloside D (25), carissaeduloside E, and carissaeduloside F) were isolated from the root bark of C. edulis (syn. C. spinarum) [94]. Out of all of these, carissaedulosides A (24) and carissaedulosides D (25) have been reported for their cytotoxic effects (Table 5) against cancer cell lines [94].

\section{Pharmacological Profile}

The traditional uses of Carissa species have inspired researchers to verify its utility through scientific pharmacological screening. Several crude extracts as well as bioactive constituents extracted from various plant parts have been evaluated for different biological activities, e.g., antioxidant, analgesic, anti-asthmatic, anticancer, anti-inflammatory, antidiabetic, antiulcer, anxiolytic, hepatoprotective, chemopreventive, hypotensive, and wound healing. Their medicinal potential has been observed in various animal models (in vitro as well as in vivo), scientifically proving the traditional utilisation of this plant.

\subsection{Antioxidant Activity}

Antioxidants are vital molecules that protect the body from harmful effects caused by free radicals. Their consumption reduces the mortality and morbidity caused by degenerative diseases $[113,114]$. Different assays successfully used by the researchers to analyse the antioxidant capacity in plants are the 2,2-azino-bis(3-ethylbenzthiazoline-6sulphonic acid) free radical assay (ABTS), ferric reducing antioxidant power assay (FRAP), 2,2-diphenyl- $\beta$-picrylhydrazyl free radical assay (DPPH), phosphomolybdenum inhibition or total antioxidant capacity (TAC) assay, $\beta$-carotene bleaching, superoxide radical scavenging activity, lipid peroxidation assay, thiobarbituric assay, hydroxyl radical, and chelating power assay.

The antioxidant properties of the Carissa species have also been assessed by the methods mentioned above, and a detailed report of antioxidant potential of Carissa species is presented in Table 3. The most frequently used in vitro assays for Carissa species were the DPPH, ABTS, FRAP, and hydrogen peroxide scavenging activity assays, where ascorbic acid, rutin, and Trolox were used as the positive control. The DPPH assay showed that among all Carissa species, maximum antioxidant potential was observed in hydroethanolic leaves' extract of C. macrocarpa $\left(\mathrm{EC}_{50} 26 \mu \mathrm{g} / \mathrm{mL}\right)$ [83,95], ethanolic leaves' extract of C. carandas $\left(\mathrm{IC}_{50} 1.292 \mu \mathrm{g} / \mathrm{mL}\right.$ ) [44], and aqueous leaves' extract of C. spinarum (syn. C. opaca) $\left(\mathrm{EC}_{50} 38 \mu \mathrm{g} / \mathrm{mL}\right)$ [74]. Similarly, the ABTS assay showed maximum antioxidant activity $\left(E_{50} 70 \mu \mathrm{g} / \mathrm{mL}\right.$ ) in the butanol fraction of methanol leaves' extract of C. spinarum (syn. C. opaca) [74] and methanol extract of leaves of C. carandas $\left(\mathrm{EC}_{50} 1.75 \mu \mathrm{g} / \mathrm{mL}\right)$ [80]. Whereas the total antioxidant assay showed maximum antioxidant activity in the aqueous fraction of leaves of C. spinarum (syn. C. opaca) $\left(\mathrm{EC}_{50} 81 \mu \mathrm{g} / \mathrm{mL}\right)$ [74]. The thiobarbituric assay showed hydroethanolic stem extract of C. macrocarpa $\left(\mathrm{EC}_{50} 3.73 \mu \mathrm{g} / \mathrm{mL}\right)$ with good antioxidant activity [83], whereas hydrogen peroxide scavenging activity revealed better 
antioxidant activity in the hexane fraction of leaves' extract of C. spinarum (syn. C. opaca) $\left(\mathrm{EC}_{50} 19 \mu \mathrm{g} / \mathrm{mL}\right)$ and the $n$-hexane extract of C. carandas $\left(\mathrm{EC}_{50} 1.802 \mu \mathrm{g} / \mathrm{mL}\right)[44,74]$. In all other assays, such as scavenging ability of superoxide radicals, scavenging ability of hydroxyl radicals, and chelating power assays, different fractions of leaves and fruits of C. spinarum (syn. C. opaca) showed maximum antioxidant potential than the other species of Carissa (Table 3).

From Table 3, it is evident that C. spinarum, C. carandas, and C. macrocarpa have lower $\mathrm{IC}_{50}$ and $\mathrm{EC}_{50}$ values, which revealed their higher antioxidant potential. Whereas, in comparison to all species, C. carandas leaves have more antioxidant potential, followed by C. macrocarpa and C. spinarum. Various previous studies suggested that the fruits and leaves of Carissa species have significant antioxidant characteristics that can be useful as a potential preventive medication against free radicals-mediated disease as well as an antioxidant drug in the pharmaceutical and food industry $[74,77,115]$.

Among all tested compounds for in vitro antioxidant activity, carissanol (5), carinol (6), and naringin (19), isolated from stem and leaves of C. spinarum and C. carandas by Wangteeraprasert et al. [23] and El-Desoky et al. [70] respectively, have shown good antioxidant activities. Similarly, olivil (18) ( $\left.\mathrm{IC}_{50} 18.1 \mu \mathrm{M}\right)$ and carinol (6) $\left(\mathrm{IC}_{50} 20.2 \mu \mathrm{M}\right)$ isolated from C. spinarum leaves revealed the highest antioxidant activity through the DPPH assay [19], whereas naringin (19) from C. carandas leaves also showed significant antioxidant potential $\left(\mathrm{EC}_{50} 11.2 \mu \mathrm{M}\right)$ [67]. The literature shows that all studied Carissa species have strong in vitro antioxidant potential; however, in vivo studies need to validate the antioxidant potency of the genus.

\subsection{Antimicrobial Activity}

Various crude extracts and isolated compounds from different natural resources, especially from plants, have always been observed as a rich source of chemical compounds for controlling bacterial and fungal infections. Different assays that have been used in the literature for the screening of plant extracts for the antimicrobial potential are the Agar disk diffusion assay, Agar dilution assay, broth microdilution assay, and minimum inhibitory concentration (MIC) assay [116]. MIC was proposed to be the most prominent and accurate method to check microbe (bacteria/fungi) resistance to an antimicrobial drug or agent [117].

The overview of reported antimicrobial assays of leaves, stem, and root extracts of different Carissa species against Gram-negative and Gram-positive bacterial strains and some fungal human pathogens is presented in Table 4. Previous studies revealed good antibacterial activity (in vitro) of all plant parts (leaves, fruits, and roots) of Carissa species against human pathogens such as Bacillus subtilis, Pseudomonas aeruginosa, Enterococcus faecalis, Escherichia coli, Klebsiella pneumoniae [35,46,88], Salmonella typhi [76], Candida albicans $[7,22,34,89]$, and Streptococcus pyogenes [22,34]. The root extract of C. spinarum was found most active against $P$. aeruginosa at MIC of $8.0 \mu \mathrm{g} / \mathrm{mL}$ [35], and Staphylococcus aureus at MIC of $312 \mu \mathrm{g} / \mathrm{mL}$ [118]. In contrast, fruits extract of C. carandas showed good activity against K. pneumoniae and S. aureus, with the same MIC of $0.3125 \mathrm{mg} / \mathrm{mL}$ [87]. Similarly, $n$-butanol fraction from the root and leaves' extracts of C. macrocarpa (syn. C. grandiflora) showed maximum antibacterial activity against $S$. epidermidis with MIC of 0.24 (roots) and $0.56 \mathrm{mg} / \mathrm{mL}$ (leaves), and S. aureus with MIC of 0.82 (roots) and $0.67 \mathrm{mg} / \mathrm{mL}$ (leaves) [89].

The essential oil of the stem of $C$. macrocarpa has also shown good antimicrobial activity against Salmonella enterica and B. subtilis (MIC $0.46 \mathrm{mg} / \mathrm{mL}$ ) [7]. The overall comparative study shows that $C$. carandas and $C$. spinarum are most effective against bacterial pathogens. Whereas, against fungal pathogens (Alternaria solani, C. albicans, Aspergillus flavus, and Penicillium monotricale), only the ethyl acetate fraction from C. spinarum root extract (syn. C. opaca) (MIC $7.8 \mu \mathrm{g} / \mathrm{mL}$ ) and essential oil from the fruits of C. macrocarpa (MIC 0.46 $\mathrm{mg} / \mathrm{mL}$ ) have been screened by Awasthi et al. [34] and Souilem et al. [7]. No data are available on the antifungal activity of other Carissa species. 
Additionally, ursolic acid (2) and $3 \beta$-hydroxyolean-11-en-28,13 $\beta$-olide (14) (from C. macrocarpa leaves and fruits, respectively) [8], and dehydrocarissone (15), carindone (from the wood of C. spinarum) [1], 2-hydroxyacetophenone (13), carinol (6), and carissone (3) (from the root of C. spinarum) [39] have also been reported for their antibacterial activity. The compound, $3 \beta$-hydroxyolean-11-en-28,13 $\beta$-olide (14), was found most active towards S. aureus, E. coli, and P. aeruginosa with MIC value of $0.06 \mathrm{mg} / \mathrm{mL}$ [8]. The above literature with quantitative data in terms of low MIC values supported the broad-spectrum antibacterial potential of Carissa species; however, the antifungal potential of Carissa species needs to be explored.

\subsection{Anticancer Activity}

The anticancer potential of crude extracts and purified constituents of Carissa species has been observed by various researchers on different cell lines, such as human cervical (Hela) cell line, breast cancer (MCF-7), hepatocellular carcinoma (HepG2), bone sarcoma (MG-63), ovarian cell (OVCAR-5), prostate cell (PC-3), human lung cancer (A549), human leukaemia (HL-60), colon cancer (SW480), and normal human (WI38) cell lines (Table 5). The root extract of $C$. spinarum showed significant anticancer activity $\left(\mathrm{IC}_{50} 34.58 \mu \mathrm{g} / \mathrm{mL}\right.$ for leukaemia HL-60; GI $5018.1 \mu \mathrm{g} / \mathrm{mL}$ for breast cancer) [93,98]. Likewise, $n$-hexane unripe fruit extract of $C$. carandas also showed significant anticancer activity $\left(E_{50} 2.492 \mu \mathrm{g} / \mathrm{mL}\right)$ for lung cancer cell lines [97,115]. Simultaneously, methanol fruit extract of $C$. carandas was observed as the most active against breast cancer, with an $\mathrm{IC}_{50}$ of $56.72 \mu \mathrm{g} / \mathrm{mL}$ [90].

Carandinol (17), isolated from C. carandas leaves, showed better anticancer activity against cervical, prostate, and normal mouse fibroblast cancer cell lines. Carandinol (17) from C. carandas also showed prominent anticancer properties towards human cervical cancer, with an $\mathrm{IC}_{50}$ of $6.87 \mu \mathrm{M}$ [41]. Sarhamnoloside (22), a constituent from C. spinarum root bark (syn. C. edulis), showed strong cytotoxic potency against human leukaemia cell lines $\left(\mathrm{IC}_{50} 0.029 \mu \mathrm{M}\right)$, lung cancer $\left(\mathrm{IC}_{50} 0.023 \mu \mathrm{M}\right)$, breast cancer $\left(\mathrm{IC}_{50} 0.025 \mu \mathrm{M}\right)$, and colon cancer $\left(\mathrm{IC}_{50} 0.137 \mu \mathrm{M}\right)$ [94]. Similarly, carissaeduloside A (24), D (25), and J (26) isolated from C. spinarum (syn. C. edulis) were screened for cytotoxic potential using human leukaemia, lung cancer, breast cancer, and colon cancer cell lines. Among these, carissaeduloside J (26) was observed with the best anticancer activity against lung cancer $(3.87 \mu \mathrm{M})$ and breast cancer $(9.231 \mu \mathrm{M})$. The anticancer activity of other compounds is shown in Table 5. Among all the tested extracts, hexane extract of unripe fruits of C. carandas showed the highest anticancer activity against lung cancer [97].

Numerous researchers have reported the anticancer potential of Carissa species against various cell lines. However, further investigation needs to trace the mechanism of action of extracts/compounds against cancer cell lines.

\subsection{Antiplasmoidal and Antimalarial Activity}

C. spinarum and C. carandas are the only two species that have been screened for antimalarial and antiplasmoidal activities. Kebenei et al. [118] revealed that the methanolic extract of the C. spinarum root bark (syn. C. edulis) exhibited significant antiplasmoidal activity $\left(\mathrm{IC}_{50} 1.95 \mathrm{mg} / \mathrm{mL}\right.$ ) against D6 strains (chloroquine-sensitive) of Plasmodium falciparum parasite, which could be due to the presence of nortrachelogenin (23) $\left(\mathrm{IC}_{50} 14.50 \mu \mathrm{g} / \mathrm{mL}\right.$ ). According to the authors, the crude extract of the root bark of $C$. spinarum was a good and easily available source for the development of an antimalarial drug $[119,120]$. Similarly, Bapna et al. [120], through their in vitro study on P. falciparum 3D7 strains, stated higher antimicrobial potential of the methanol extracts of leaves, stem, bark, and fruits from C. carandas $\left(\mathrm{IC}_{50} 13.57-69.63 \mu \mathrm{g} / \mathrm{mL}\right.$ ), in contrast to aqueous extracts $\left(\mathrm{IC}_{50} 41.52-100 \mu \mathrm{g} / \mathrm{mL}\right.$ ). The authors used the 3-[4,5-dimethylthiazol-2-yl]-2,5-diphenyltetrazolium bromide (MTT) assay to analyse host cell cytotoxicity on the Madin-Darby Canine Kidney (MDCK) cell line and observed no cytotoxicity in the maximum tested dose.

Gebrehiwot et al. [120] screened the hydro-alcoholic and chloroform root extracts of C. spinarum for antiplasmoidal activity. They observed that both extracts exhibited signifi- 
cant activity $(p<0.05)$ at $400 \mathrm{mg} / \mathrm{kg}$ against $P$. berghei in Swiss albino mice $(43.23 \% \pm 0.66 \%$ parasitic suppression at day hour with hydroalcoholic extract and 51.64\% $\pm 2.13 \%$ inhibition with chloroform extract), where chloroquine phosphate was used as a positive control $(51.82 \% \pm 0.72 \%$ parasitic suppression). The authors also observed that plant extract-treated mice did not exhibit any acute toxic effects up to $2000 \mathrm{mg} / \mathrm{kg}$.

These data support the ethnopharmacological information about the antimalarial activity of Carissa species. The in vivo assays revealed the antimalarial property at $400 \mathrm{mg} / \mathrm{kg}$ of the extract, and according to Gertsch [121], for the in vivo assay, a significant concentration should be $>200 \mu \mathrm{g} / \mathrm{mL}$. Therefore, more investigations are required to develop clinical trials that can prove the efficacy of C. spinarum and C. carandas in humans to treat malaria.

\subsection{Antiviral Activity}

Only two species of Carissa viz., C. spinarum (root bark) and C. carandas (fruits), have been explored for antiviral activity by Tolo et al. [106,122] and Arif et al. [12], respectively. Tolo et al. [122] observed significant in vitro antiviral activity $(p<0.05)$ of $C$. spinarum (syn. C. edulis) root extract at $1 \mathrm{mg} / \mathrm{mL}$ against herpes simplex viruses HSV-I (EC $\left.\mathrm{EC}_{50} 15.1 \pm 0.57 \mu \mathrm{g} / \mathrm{mL}\right)$, HSV-II $\left(E_{50} 6.9 \pm 1.27 \mu \mathrm{g} / \mathrm{mL}\right)$, HSV-I APr $\left(\right.$ EC $\left._{50} 8.1 \pm 1.56 \mu \mathrm{g} / \mathrm{mL}\right)$, and HSV-I TK- $\left(\mathrm{EC}_{50}\right.$ $11.1 \pm 5.66 \mu \mathrm{g} / \mathrm{mL}$ ), in comparison to the significant antiviral activity of Acyclovir (positive control) against HSV-I (EC $500.91 \pm 0.46 \mu \mathrm{g} / \mathrm{mL}), \mathrm{HSV}-\mathrm{II}\left(\mathrm{EC}_{50} 0.87 \pm 0.44 \mu \mathrm{g} / \mathrm{mL}\right), \mathrm{HSV}-\mathrm{I}$ $\mathrm{AP}^{\mathrm{r}}\left(\mathrm{EC}_{50}>5.0 \mu \mathrm{g} / \mathrm{mL}\right)$, and HSV-I TK$-\left(\mathrm{EC}_{50}>5.0 \mu \mathrm{g} / \mathrm{mL}\right)$. According to Tolo et al. [123], $250 \mathrm{mg} / \mathrm{kg}$ roots' extract of C. spinarum (aqueous) possessed good anti-herpes simplex virus activity in comparison to acyclovir $(5 \mathrm{mg} / \mathrm{kg})$; as a result, it may be used for developing an effective treatment against HSV infections.

Tolo et al. [106] also screened various phytocompounds, such as lupeol (1), oleuropein (20), carissol (12), and $\alpha$-amyrin (21), from the C. spinarum root bark for antiviral activity (in vitro) against Herpes simplex virus strains 1 (7401H HSV-1, TK- B2006 HSV-1, APr $7401 \mathrm{H}$ HSV-1), up to $1 \mathrm{mg} / \mathrm{mL}$ concentration. The study revealed a significant activity $(p<0.05)$ of Lupeol (1) for both sensitive and resistant strains $\left(\mathrm{EC}_{50} 2.98 \mu \mathrm{g} / \mathrm{mL}\right.$ for $7401 \mathrm{H}$ HSV-1; $\mathrm{EC}_{50} 3.66 \mu \mathrm{g} / \mathrm{mL}$ for APr 7401H HSV-1; $\mathrm{EC}_{50} 4.2 \mu \mathrm{g} / \mathrm{mL}^{\mathrm{H}}$ for TK $\left.\mathrm{TK}^{-} \mathrm{B} 2006 \mathrm{HSV}-1\right)$, with a high therapeutic index $(\mathrm{TI}>38)$ similar to the positive control $\left(\mathrm{EC}_{50} 0.45 \mu \mathrm{g} / \mathrm{mL}\right.$ for 7401H HSV-1; EC $50>10 \mu \mathrm{g} / \mathrm{mL}$ for APr $7401 \mathrm{H}$ HSV-1; $\mathrm{EC}_{50}>10 \mu \mathrm{g} / \mathrm{mL}$ for TK-B2006 HSV-1). According to the authors, the compound at $10 \mu \mathrm{g} / \mathrm{mL}$ was found as virucidal, as evident in E6 cells by $98.3 \%$ (in vitro). Arif et al. [12] explored the effectiveness of $C$. carandas fruits' ethanolic extract $(6,3$, and $12 \mu \mathrm{g} / \mathrm{mL})$ for antiviral activity against Poliovirus HIV-1, Sindbis virus, and Herpes simplex virus, respectively.

Whereas, for the in vivo study, C. spinarum (syn. C. edulis) at $250 \mathrm{mg} / \mathrm{kg}$ showed significant antiviral activity (delayed infection by two days) against $7401 \mathrm{H}$ HSV-1-infected Balb/C mice (female), and similar results were obtained with the $5 \mathrm{mg} / \mathrm{kg}$ positive control. At $250 \mathrm{mg} / \mathrm{kg}$, mice showed no signs of toxic effects.

From the above studies, it has been made clear that Carissa species have significant activity towards different Herpes simplex viral strains due to the presence of lupeol (1). The members of this genus can also be used to formulate effective drugs against other types of viruses.

\subsection{Anticonvulsant Activity}

Anticonvulsant activity was only observed with C. spinarum and C. carandas. All studies suggested biologically active constituents in the root bark of C. spinarum and C. carandas that have anticonvulsant activity. The anticonvulsant activity of different fractions (aqueous, $n$-butanol, and ethyl acetate) obtained from hydro-alcoholic $(60 \% v / v)$ root bark extract of C. spinarum (syn. C. edulis) was observed against maximal electroshock (MES) in chicks, pentylenetetrazole (PTZ), and 4-aminopyridine-induced seizure (90 and $15 \mathrm{mg} / \mathrm{kg}$, respectively) in mice at dose levels between 1.25 and $10 \mathrm{mg} / \mathrm{kg}$ by Jamilu et al. [124]. The researchers observed $80 \%$ anticonvulsant activity in the aqueous fraction $(10 \mathrm{mg} / \mathrm{kg})$ in PTZ-induced seizures as compared to valproate $(200 \mathrm{mg} / \mathrm{kg})$, a standard antiepileptic 
drug that showed $100 \%$ protection. Whereas, ethyl acetate and $n$-butanol fractions also showed protection, but that was not dose-dependent. In MES-induced seizures in chicks, $30 \%$ protection was observed with an ethyl acetate fraction at $2.5 \mathrm{mg} / \mathrm{kg}$, and according to the authors, the entire fractions reduced the mortality rate in a dose-independent manner. Thus, it was observed that all used fractions of C. spinarum have anticonvulsant activity, particularly against MET- and PTZ-induced seizures.

Yau et al. [5] investigated the $\mathrm{LD}_{50}$ of C. spinarum (syn. C. edulis) root bark extract on convulsions induced by pentylenetetrazole (PTZ) and maximal electroshock (MES) (in mice and chicks, respectively) through oral and intraperitoneal administration. The $\mathrm{LD}_{50}$ values of 282.8 (intraperitoneal) and $5000 \mathrm{mg} / \mathrm{kg}$ (oral) were observed for C. edulis root extract. Further, the extract exhibited $40 \%$ and $20 \%$ inhibition of convulsions in mice induced by PRZ at 20 and $5 \mathrm{~kg} / \mathrm{mL}$ respectively, as compared to benzodiazepine (100\% inhibition). On MES-induced convulsions in chicks, root extract showed $90 \%$ inhibition compared to $100 \%$ protection with $20 \mathrm{mg} / \mathrm{kg}$ of phenytoin. In the year 2009 , the anticonvulsant effect of a root bark ethanolic extract from C. carandas was studied by Hegde et al. [21] on electrically, picrotoxin, chemically, $\mathrm{N}$-methyl-dl-aspartic acid, pentylenetetrazole, and bicuculline-induced seizures in a mouse model. The study showed that ethanolic extract $(100-400 \mathrm{mg} / \mathrm{kg})$ reduced $(p<0.001)$ the duration of seizures induced by MES. At 100 , 200 , and $400 \mathrm{mg} / \mathrm{kg}$ of extract, $25 \%, 50 \%$, and $62.5 \%$ inhibition of seizures was observed. Whereas in another case, the seizures were induced with pentylenetetrazole, and similar doses showed significant protection and delayed progression of tonic seizures formed by $\mathrm{N}$-methyl-dl-aspartic acid and picrotoxin. On the contrary, the extract showed an insignificant effect against bicuculline-produced seizures. From the above information, it is clear that $C$. spinarum and C. carandas roots exerted significant anticonvulsant effects in in vivo studies, and therefore, both species can be exploited for the treatment of epilepsy.

\subsection{Antinociceptive Activity}

The methanolic extract from C. carandas leaves $(50,100,200$, and $400 \mathrm{mg} / \mathrm{kg}$ ) exhibited significant antinociceptive activity using the Swiss albino mice gastric pain model (acetic acid as an inducer). It decreased the number of writhings as compared to the antinociceptive standard drug aspirin (at 200 and $400 \mathrm{mg} / \mathrm{kg}$ ) [123]. In another study, the methanolic extract from C. spinarum (syn. C. edulis) leaves reduced $47.04-47.19 \%$ and $38.96-89.26 \%$ of pain in rats in the early and late phase respectively, at $100 \mathrm{mg} / \mathrm{kg}(47.04 \%)$ and $150 \mathrm{mg} / \mathrm{kg}$ ( $47.19 \%)$ body weight. However, methanol root bark extracts reduced it by $21.5-41.89 \%$ (early phase) and 21.4-90.62\% (late phase) in comparison to diclofenac $(15 \mathrm{mg} / \mathrm{kg}$, standard drug), which reduced the pain by $27.37-34.9 \%$ and $88.24-90.28 \%$ in the early and late phase, respectively [125]. Mworia et al. [126] observed a 73.77\% and $86.89 \%$ reduction in acetic acid-associated pain in a mouse model by using acetone leaves' extract of $C$. spinarum (50 and $100 \mathrm{mg} / \mathrm{kg}$ body weight, respectively), in comparison to diclofenac sodium (70.49\% at $15 \mathrm{mg} / \mathrm{kg})$. Similarly, Parvin [20] also observed significant $(p<0.01)$ antinociceptive activity in Swiss albino mice with C. carandas (leaves' methanolic extract) at 200 and $400 \mathrm{mg} / \mathrm{kg}$ body weight, as compared to the standard drug (diclofenac, $1 \mathrm{mg} / \mathrm{kg}$ ). Traditionally, Carissa plants have been reported in different forms such as decoction, infusion, etc., to treat fever and pain, and these in vivo studies provide evidence for the antinociceptive effect of the Carissa species.

\subsection{Antidiabetic Activity}

The ethanolic leaves' extract from C. spinarum (syn. C. edulis) $(2 \mathrm{~g} / \mathrm{kg})$ was observed with significant antidiabetic activity in comparison with the reference drugs (metformin (500 mg/ $\mathrm{kg}$ ) and $3 \mathrm{mg} / \mathrm{kg}$ of glibenclamide) in diabetic adult male albino rats [127]. Swami et al. [128] observed that an aqueous extract from C. carandas at 500 and $1000 \mathrm{mg} / \mathrm{kg}$ showed antidiabetic activity $(p<0.05)$ by decreasing the blood glucose levels in diabetic Wistar rats (alloxan-induced model). Furthermore, the authors observed that methanol extracts also lowered the elevated levels of blood glucose significantly $(p<0.001)$ after 
$24 \mathrm{~h}$ at $400 \mathrm{mg} / \mathrm{kg}$ orally, as compared to the control. This was attributed to the total polyphenols and total flavonoids content of the plant. Itankar et al. [129] observed a decrease in the level of blood glucose ( $48 \%$ and $64.5 \%$ ) in Wistar rats after using $400 \mathrm{mg} / \mathrm{kg}$ per oral methanol extract of unripe C. carandas fruit extract and its fraction (ethyl acetate), respectively. The authors also revealed the higher antidiabetic potential in the case of ethyl acetate fraction, in contrast to methanol extract. They concluded that fractionation increased polymerisation/segregation of biologically active metabolites.

On the other hand, Madhuri and Neelagund [130] stated that the aqueous extract from C. carandas fruits showed potent inhibition of $\beta$-glucosidase activity between 1.25 and $10 \mathrm{mg} / \mathrm{mL}$ concentration.

These data also support the traditional and practical information about the antidiabetic activity of Carissa species. Leaves and fruit extracts of $C$. spinarum and C. carandas significantly inhibit the blood glucose level and can be used to isolate bioactive constituents in drug preparations for human welfare.

\subsection{Antipyretic Activity}

Various researchers observed the antipyretic activity of leaves and roots of the Carissa genus and therefore validated its folk use in the treatment of pain and fever. Hegde and Joshi [57] observed that the ethanolic root extract from C. spinarum (100, 200, and $400 \mathrm{mg} / \mathrm{kg}$ ) reduced the body temperature in Wistar rats (Brewer's yeast-induced pyrexia), and therefore stated the significant activity $(p<0.05)$ of ethanolic extract. Garg et al. [131] also showed the significant antipyretic activity $(p<0.01)$ of aqueous extract of $C$. carandas leaves at all dose levels $(100,200$, and $400 \mathrm{mg} / \mathrm{kg}$ ) compared to $200 \mathrm{mg} / \mathrm{kg}$ of paracetamol. Hati et al. [132] and Bhaskar and Balakrishnan [133] also stated that methanolic extracts of C. carandas leaves and roots at 100 and $200 \mathrm{mg} / \mathrm{kg}$ reduced $(p<0.01)$ pyrexia in the albino rats in a dose-dependent manner. According to Gitahi [134], the leaves and the root bark extract (dichloromethane:methanol) of C. spinarum (syn. C. edulis) have profound antipyretic activities compared to the drug $(100 \mathrm{mg} / \mathrm{kg}$, aspirin) at a concentration of 50, 100, and $150 \mathrm{mg} / \mathrm{kg}$. On the other hand, Allam et al. [135] also showed the highest antipyretic potential of the crude methanolic extract and butanol fraction of the aerial parts (leaves and stem) of C. macrocarpa at $100 \mathrm{mg} / \mathrm{kg}$ in a yeast-induced hyperpyrexia model, in male albino rats. Acetylsalicylic acid $(100 \mathrm{mg} / \mathrm{kg})$ was used as a standard drug.

The antipyretic data show that the authors have used a high concentration of extracts for the experiments; therefore, further in vivo studies need to validate the traditional use of different parts of the genus Carissa.

\subsection{Anti-Inflammatory Activity}

The different plant parts of the genus Carissa such as roots, stem, and leaves have been screened for anti-inflammatory activity by various researchers. Bhaskar and Balakrishnan [133] screened the root extracts from C. carandas (ethanol and aqueous) for anti-inflammatory activity. It was found that both the extracts significantly reduced the formation of oedema induced by carrageenan after $2 \mathrm{~h}$. Anupama et al. [42] reported the anti-inflammatory activity of methanol extract from $C$. carandas fruits using a carrageenaninduced hind paw oedema model. The extract at all dose levels $(100,200$, and $400 \mathrm{mg} / \mathrm{kg})$ showed significant activity when administered orally to the experimental rats as compared with $50 \mathrm{mg} / \mathrm{kg}$ of indomethacin. Hati et al. [132] studied the effect of $C$. carandas leaf methanolic extract $(200 \mathrm{mg} / \mathrm{kg}$ ) against dextran, histamine, and carrageenan-induced paw oedema, and observed $71.90 \%, 72.10 \%$, and $71.80 \%$ inhibition respectively, at the end of $3 \mathrm{~h}$.

Beck and Namdeo [136] studied the anti-inflammatory activity of different extracts from C. spinarum leaves (petroleum ether, chloroform, alcoholic, and aqueous) using a formalin-induced rat paw oedema model in rats. The extracts at $200 \mathrm{mg} / \mathrm{kg}$ exhibited anti-inflammatory activity $(p<0.01)$ as compared to the control metamizole $(20 \mathrm{mg} / \mathrm{kg})$. Among the extracts, the aqueous extract was found to be the most potent as compared to other extracts tested. Yau et al. [137] evaluated the anti-inflammatory effects of a residual 
aqueous fraction from the C. spinarum (syn. C. edulis) root bark ethanolic extract using a carrageenan-induced paw oedema model in rats. It was found that the residual fraction at $600 \mathrm{mg} / \mathrm{kg}$ and standard ketoprofen at $10 \mathrm{mg} / \mathrm{kg}$ showed a decrease $(p<0.05)$ in oedema up to $4 \mathrm{~h}$. According to Woode et al. [138], anti-inflammatory activities (in vivo) in the alcoholic extract of C. spinarum (syn. C. edulis) roots could be due to the presence of antioxidants.

Saher et al. [139] observed the anti-inflammatory activity of C. carandas fruits (immature, mature, and ripe) using carrageenan-induced paw oedema and cotton pellets-induced granuloma in Wistar rats. The highest percentage inhibition was observed with mature fruit extract $(200 \mathrm{mg} / \mathrm{kg}$ ) at $3 \mathrm{~h}$. Whereas, at a $100 \mathrm{mg} / \mathrm{kg}$ dosage, immature fruit extract exhibited $68 \%$ (highest) inhibition in the carrageenan-induced model compared to mature and ripe fruit extracts after $3 \mathrm{~h}$. On the other hand, in the cotton pellet method, less than $50 \%$ inhibition of granuloma was found with the immature $(21.93 \%)$, ripe $(20.14 \%)$, and mature $(24.91 \%)$ fruit extracts compared to standard diclofenac sodium (45\%). Allam et al. [135] screened the ethyl acetate and dichloromethane fractions from crude methanolic extract (leaves and stem) of C. macrocarpa aerial parts for anti-inflammatory activity and observed maximum activity at $100 \mathrm{mg} / \mathrm{kg}$, with $43 \%$ and $41 \%$ inhibition respectively, in comparison with the standard drug indomethacin $(47 \%$ at $10 \mathrm{mg} / \mathrm{kg})$.

In case of isolated compounds, the anti-inflammatory potential of naringin (19) from C. carandas leaves at $50 \mathrm{mg} / \mathrm{kg}$ showed potent inhibition (59.24\%) of inflammation when compared with the control (48.10\% with $20 \mathrm{mg} / \mathrm{kg}$ of indomethacin) [70].

On the other hand, carisssone (3) and scopoletin (8), purified from C. carandas roots, also showed inhibition of nitric oxide (NO) production ( $\mathrm{IC}_{50} 20.1 \pm 2.99$ and $24.6 \pm$ $1.36 \mu \mathrm{g} / \mathrm{mL}$, respectively) as compared to the standard inhibitor of NO (IC $5019.82 \pm$ $1.64 \mu \mathrm{g} / \mathrm{mL}$ ) with no adverse effect on cell viability [110]. It is clear from the above data and traditional information that different plant parts of $C$. spinarum and C. carandas were found to be anti-inflammatory, and their effect is related to their phytoconstituents, such as carissone (3) and naringin (19). However, the mechanistic basis of these extracts/compounds remains obscure.

\subsection{Hepatoprotective Activity}

Bhaskar and Balakrishnan [140] studied the hepatoprotective effects of the C. carandas root extracts (aqueous and ethanolic) in rats. The authors observed that both extracts at doses of 200 and $100 \mathrm{mg} / \mathrm{kg}$ exhibited significant hepatoprotection $(p<0.05)$ by reducing lipid peroxidation, serum transaminase, alkaline phosphate, and bilirubin, while elevating the serum as well as liver glutathione levels $(p<0.01)$ as compared to standard aspirin $(150 \mathrm{mg} / \mathrm{kg})$. Subsequently, Hegde and Joshi analysed the hepatoprotective effect of C. spinarum roots' ethanolic extract in rats. The authors stated that pre-treatment (orally) with extract $(100,200$, and $400 \mathrm{mg} / \mathrm{kg})$ alleviated $(p<0.01)$ the hepatotoxicity induced by $\mathrm{CCl}_{4}$ and paracetamol in a dose-dependent manner [141]. According to the authors, root extracts reduced the hepatotoxicity in rats by decreasing lipid peroxidation and bilirubin and enhancing the level of protein, glutathione, uric acid, catalase, and superoxide dismutase.

Similarly, Sahreen et al., also observed the hepatoprotective activity of methanol extract of C. spinarum leaves (syn. C. opaca) in Male Sprague-Dawley rats [58]. The authors stated that the extract at $200 \mathrm{mg} / \mathrm{kg}$ significantly reduced $\mathrm{CCl}_{4}$-induced hepatotoxicity in rats as compared to the positive control, $50 \mathrm{mg} / \mathrm{kg}$ of silymarin. It was concluded that the activity in leaves could be due to its antioxidant activity and membrane-stabilizing potential.

El-Desoky et al. evaluated the defatted aqueous methanol leaves' extract $(500 \mathrm{mg} / \mathrm{kg})$ of C. carandas for its hepatoprotective effects and compared it with the drug silymarin $(100 \mathrm{mg} / \mathrm{kg})$ [70]. The authors observed that the extract significantly $(p<0.01)$ reversed elevated serum liver marker enzymes, reduced malondialdehyde (MDA), and subsequently increased glutathione (GSH) content in liver homogenate.

The crude extracts, their fraction, and pure compounds isolated from the plants have been proven as a very effective drug for liver disease. These extracts possessed 
sufficient efficacy to treat severe liver disease caused by toxic chemicals, viruses, and excess alcohol. Thus, from the above findings, it can be stated that Carissa species such as C. spinarum and C. carandas are promising hepatoprotective agents, validating the assertion of traditional healers.

\subsection{Antiarthritic Activity}

Hegde et al. [84] observed the significant $(p<0.05)$ and dose-dependent anti-arthritic activity of ethanolic root extracts of C. spinarum (100, 200, and $400 \mathrm{mg} / \mathrm{kg}$, p.o.) and phenylbutazone (100 mg/kg, i.m.) in Freund's adjuvant-induced polyarthritis model in rats. Dar et al. [6] also studied the antiarthritic activity of ethanolic leaves' extract from C. carandas (200 and $400 \mathrm{mg} / \mathrm{kg}$ ) in arthritis model rats (adjuvant-induced) and observed that the ethanol extract showed a reduction $(p<0.01)$ in paw volume when compared with aspirin $(50 \mathrm{mg} / \mathrm{kg})$. According to the authors, this property is attributed to the synergistic potential of phytoconstituents.

The above-discussed data suggested the significant antiarthritic potential of leaves and root extracts of $C$. spinarum and $C$. carandas, which may be possibly due to lanost-5-en$3 \beta$-ol-21-oic acid (27). However, the role of other phytocompounds of the genus needs to be explored.

\subsection{Adaptogenic Activity}

Arif et al. $[87,109]$ screened the crude ethanolic extract and a lanostane triterpenoid, lanost-5-en-3 $\beta$-ol-21-oic acid (27), from the C. carandas fruit ethanolic extract (of 200, 100, and $10 \mathrm{mg} / \mathrm{kg} /$ day), and it was evaluated for the adaptogenic activity using anoxia stress tolerance, swimming endurance, and immunosuppression induced by cyclophosphamide (experimental mice) models. Aspirin $(25 \mathrm{mg} / \mathrm{kg})$ was used as the standard drug. The authors observed the great role of crude extract and lanostane triterpenoid in cyclophosphamide-treated mice with an increase in swimming endurance, anoxia stress tolerance, and normalcy of parameters such as $\mathrm{Hb}$, affected organ, $\mathrm{RBC}, \mathrm{WBC}$, and body weight ( $p<0.05$ and $p<0.01$, respectively). Therefore, the authors revealed the significant adaptogenic activity of lanostane triterpenoid and the crude extract as well.

\subsection{Effect on the Cardiovascular System and Cardioprotective Activity}

The cardioprotective activity and the effect of C. spinarum aerial parts on the cardiovascular system were evaluated by Al-Youssef and Hassan [69] and Sahreen et al. [142]. The cardiovascular effect of different extracts from C. spinarum (syn. C. edulis) aerial parts was evaluated in Wistar rats. The authors observed that ethyl acetate, petroleum ether, and aqueous extracts $(0.05 \mathrm{~g} / \mathrm{kg})$ showed an observable decline in arterial pressure $(27,27.2$, and $9.1 \mathrm{mmHg}$, respectively), whereas the extracts at $0.1 \mathrm{~g} / \mathrm{kg}$ exhibited a 34.5,36.3, and $32.7 \mathrm{mmHg}$ decline in blood pressure, respectively [64].

Subsequently, Sahreen et al. [143] observed the cardioprotective potential of different fractions (methanol, $n$-hexane, ethyl acetate) from C. spinarum (syn. C. opaca) leaf extracts in male Sprague Dawley rats whose cardiac function was altered by treating with carbon tetrachloride $\left(\mathrm{CCl}_{4}\right)$. The authors observed that all tested fractions showed protective effects against $\mathrm{CCl}_{4}$ intoxication, by normalizing the altered cardiac function and antioxidant enzymes. In addition to these, DNA damage and histopathological abnormalities were also restored by various fractions. However, further studies are required to validate the claim of the genus Carissa for their action against cardiovascular activity. Additionally, further studies need to include the mechanism of action of extracts for their cardioprotective potential using different model systems [143].

\subsection{Anthelmintic Activity}

Only two species of Carissa (C. spinarum and C. carandas) have been screened for anthelmintic activity. The first study on the anthelmintic activity of the C. carandas root bark was performed by John et al. [142]. According to the researchers, $50 \mathrm{mg} / \mathrm{mL}$ of the methanol 
extract caused paralysis on Indian earthworm, followed by the death of the worm, hence revealing anthelmintic activity of plant roots comparable with that of the standard drug albendazole $(10 \mathrm{mg} / \mathrm{mL})$. In another study, Harwansh et al. [37] stated that methanolic and chloroform extract of C. spinarum were found equally potent at $100 \mathrm{mg} / \mathrm{mL}$, as compared to piperazine citrate $(10 \mathrm{mg} / \mathrm{mL})$, which resulted in paralysis that led to the death of Pheretima posthuma. Mishra et al. [144] also observed in vitro anthelmintic activity in unripe fruits of C. carandas at doses of 50,100, and $150 \mathrm{mg} / \mathrm{mL}$ on Indian earthworms, and detected a short time of paralysis $(56.35,40$, and $22.35 \mathrm{~min}$ ) followed by the worms' death at $150 \mathrm{mg} / \mathrm{mL}$ of ethanolic, chloroform, and petroleum ether extracts respectively, when compared to piperazine citrate $(15 \mathrm{mg} / \mathrm{mL})$. These observations indicated that ethanolic extract took a shorter duration to cause paralysis when compared with unripe fruit extract. Similarly, Parvin [20] studied the anthelmintic activity of fresh juice of $C$. carandas leaves $(25,50$, and $100 \mathrm{mg} / \mathrm{mL})$ on earthworms by observing their paralysis and death time. The authors observed the paralysis and death time (minimum to maximum) in the range of approximately 4 to $7 \mathrm{~min}$ respectively, compared to the standard drug albendazole $(100 \mathrm{mg} / \mathrm{mL} ; 3-10 \mathrm{~min})$. The authors stated that the fresh juice of leaves showed potent anthelmintic activity in earthworms.

\subsection{Antiemetic Activity}

Mohtasheemul et al. [145] also studied and compared the antiemetic activity of C. carandas fruits and the reference drug domperidone $(100 \mathrm{mg} / \mathrm{kg})$ on a chick emetic model. Their results showed that the extract decreased the number of copper-sulphate pentahydrateinduced retches ( $50 \mathrm{mg} / \mathrm{kg}$ body weight, orally) in chicks. However, other members of the genus Carissa also need to be investigated for their anthelmintic and antiemetic potential. Additionally, studies need to be designed based on the effect of active compounds of plant extracts on the death of pathogenic worms.

\subsection{Neuropharmacological and Diuretic Activity}

Various researchers observed the neuropharmacological and diuretic activity of leaves and root bark extracts. Saha et al., studied the methanol extract of $C$. carandas to evaluate neuropharmacological and diuretic activities on male albino rats [146]. Their findings revealed significant $(p<0.01)$ neuropharmacological activity of the extract $(250$ and $500 \mathrm{mg} / \mathrm{kg}$ ). The diuretic activity of the extract was evident from the 1.46 to 1.43 reduction in the ratio of $\mathrm{Na}^{+} / \mathrm{K}^{+}$excretion at 200 and $400 \mathrm{mg} / \mathrm{kg}$ respectively, when compared to the standard diuretic furosemide $(1.48,0.5 \mathrm{mg} / \mathrm{kg})$.

The neuroprotective effect of the aqueous extract on the C. spinarum (syn. C. edulis) leaves was also evaluated by Yadang et al. [147] using the novel object recognition, Tmaze methods in mice to identify memory, learning, open-field locomotion test, along with brain acetylcholinesterase enzyme (AChE) activity. In addition to these, the authors also examined oxidative stress through different parameters such as malondialdehyde (MDA) level, glutathione, and catalase activity. The results showed that at different dose levels, such as $62.8,143,314$, and $628 \mathrm{mg} / \mathrm{kg}$, plant extract (orally administrated) increased the memory, object recognition, and also improved the locomotion of mice. Whereas Scopolamine ( $1 \mathrm{mg} / \mathrm{kg}$ body weight) administration for seven days of treatment showed a decrease in learning and memory enhancement in mice. On the other hand, mice with aqueous extract decreased the AChE activity $(2.55 \pm 0: 10 \mathrm{~mol} / \mathrm{min} / \mathrm{g})$ and brain oxidative stress. The authors concluded that by reducing AChE activity, aqueous extract enhanced the memory of mice.

Nedi et al. [148] observed the significant $(p<0.01)$ diuretic property of an extract from the C. spinarum (syn. C. edulis) root bark at $1000 \mathrm{mg} / \mathrm{kg}$, and wood maceration $(50 \mathrm{mg} / \mathrm{kg}$ ). According to the authors, the plant extract contains compounds that mediated the diuretic effect by significantly increasing the volume of urine and also enhanced the number of electrolytes, $\mathrm{K}^{+}, \mathrm{Na}^{+}$, and $\mathrm{Cl}^{-}$ions. AI-Youssef and Hassan [68] compared the diuretic potential of different extracts (petroleum ether, ethyl acetate, chloroform, and aqueous) 
from C. spinarum aerial parts at a dose of $1 \mathrm{~g} / \mathrm{kg}$, in contrast to the control (normal saline). Their result showed that petroleum ether and ethyl acetate extract $(1 \mathrm{~g} / \mathrm{kg})$ slightly affected urine output, with $9.1 \%$ and $12.7 \%$ respectively, while chloroform and aqueous extracts at the same dose significantly increased urine output by $54.5 \%$ and $45.4 \%$, respectively. Kebamo et al. [149] observed the significant diuretic activity of an aqueous fraction from C. spinarum (syn. C. edulis) root bark methanolic extract at doses of 50,500, and $1000 \mathrm{mg} / \mathrm{kg}$ in normal Wistar rats. On the other hand, n-butanol and petroleum ether fractions were devoid of activity as compared with standard hydrochlorothiazide (10 mg/ kg).

The concentration of extracts used in the above-discussed studies was relatively high. Furthermore, in vivo studies are required to validate the utilisation of Carissa species for the neuropharmacological and diuretic properties.

\subsection{Wound Healing Activity and Toxicological Study}

Sanwal and Chaudhary [150] applied cold macerated $1 \%$ and $2.5 \%$ methanolic extract of C. spinarum root extract against a burn wound mice model and observed wound contraction and epithelisation, therefore proving the significant wound healing potential of the root extract. Subsequently, the in vivo toxicity (acute as well as subacute) of the root extract of C. spinarum in Swiss albino mice was evaluated by Gebrehiwot [151]. According to the researchers, the hydro-methanolic and chloroform extracts at a $5000 \mathrm{mg} / \mathrm{kg}$ dose did not produce significant physical and behaviour changes, and no death was recorded. Whereas, in sub-acute toxicity studies, the extracts showed an insignificant change $(p>0.05)$ of haematological and physical parameters in the treated groups when associated with the control groups. Shamim also studied the acute, subacute, and sub-chronic toxicological studies of the ethanolic extracts of $C$. carandas leaves. The authors reported that the extracts at 1750 and $5000 \mathrm{mg} / \mathrm{kg}$ did not exhibit any mortality in the acute toxicity evaluation, whereas subacute toxicity exhibited no signs of toxicity and mortality in the treated group, contrary to the control ones at $5000 \mathrm{mg} / \mathrm{kg}$ [152]. On the other hand, chronic toxicity $(5000 \mathrm{mg} / \mathrm{kg}$ ) showed some changes in the histological parameters. The ethanolic extract of C. spinarum roots at $2000 \mathrm{mg} / \mathrm{kg}$ exhibited no toxicity or behavioural changes in Wistar albino rats during 14 days of treatment [57]. They have an important regulatory role and are therefore seen as therapeutic goals of Carissa species to control the wound healing processes in the future.

The safety evaluation of C. carandas extract $(5000 \mathrm{mg} / \mathrm{kg})$ was evaluated by Bhaskar and Balakrishnan [133]. According to the authors, this dose was tolerated by rats, and no adverse symptoms or deaths have been observed in acute toxicity investigation. However, an oral $\mathrm{LD}_{50}$ of the extract was found unascertainable in rats ( $>5000 \mathrm{mg} / \mathrm{kg}$ body weight). According to the authors, the plant extract is considered non-toxic if oral LD $_{50}$ values are higher than $4 \mathrm{~g} / \mathrm{kg}[153,154]$. Recently, an in vivo acute toxicity assay of C. spinarum (syn. C. edulis) extracts (methanol and methanol:water) at doses of 50-2000 mg/kg showed that there was no behavioural change or death observed during seven days of treatment [91]. According to the authors, both extracts were found safe at doses of up to $2000 \mathrm{mg} / \mathrm{kg}$ body weight in mice. Although the literature proved the wound healing effect of the root extract of C. spinarum, still more investigations are required. Toxicity studies that were performed on Carissa species also showed non-toxicity of C. spinarum and C. carandas extracts up to $5000 \mathrm{mg} / \mathrm{kg}$. Dossou-Yovo et al. [155] also proved the safety of the C. spinarum roots' hydroalcoholic extract at 500,1000, and $5000 \mathrm{mg} / \mathrm{kg}$ by conducting acute and subacute oral toxicity on Wistar rats through the oral route.

\section{Conclusions and Summary}

Carissa genus is a key contributor of valuable phytoconstituents, and various Carissa species have been screened for their nutrients, bioactive constituents, and for pharmaceutical aspects, which are important for maintaining good health. The present review is focused on the research carried out on various aspects of only four species of Carissa (C. spinarum, C. carandas, C. macrocarpa, and C. bispinosa). The other species reported in the literature, 
such as C. opaca, C. edulis, C. lanceolata, C. congesta, and C. grandiflora, are the synonyms of C. spinarum and C. macrocarpa, respectively. A total of 121 compounds ( 35 polyphenols (phenolic acids and flavonoids), 30 lignans, 41 terpenoids, 7 steroids, 2 coumarins, and 6 cardiac glycosides) have been extracted from C. spinarum, C. carandas, and C. macrocarpa. No report is available on the isolation of chemical constituents from C. bispinosa. Among all these compounds, only a few compounds have been screened/tested for different biological activities. For example, lupeol (1), oleuropein (20), carissol (12), and $\alpha$-amyrin (21), extracted from C. spinarum, have been tested against herpes simplex viruses (I, II, III), naringin (19), carisssone (3), and scopoletin (8), isolated from C. carandas, have been checked only for anti-inflammatory activity, and Carandinol (17), sarhamnoloside (22), and carissaeduloside A (24), D (25), and J (26), from C. carandas and C. spinarum respectively, were explored for anticancer activity. Similarly, carissanol (5) and naringin (19), isolated from C. spinarum and C. carandas, and olivil (18) and carinol (6) isolated from C. spinarum have shown good antioxidant activities. Besides these, $3 \beta$-hydroxyolean-11-en-28,13 $\beta$-oilde (14) and ursolic acid (2), isolated from C. macrocarpa, and carinol (6) and carissone (3) from C. spinarum have been evaluated for antimicrobial activities.

Among all the Carissa species, most of the pharmacological activities were tested on C. spinarum and C. carandas, whereas C. macrocarpa was used to evaluate antioxidant, antimicrobial, anticancer, antipyretic, and anti-inflammatory activities. The most extensively used parts from various reported activities were the roots and leaves of $C$. spinarum and C. carandas, and the stem and flowers of C. macrocarpa (Figure 4).

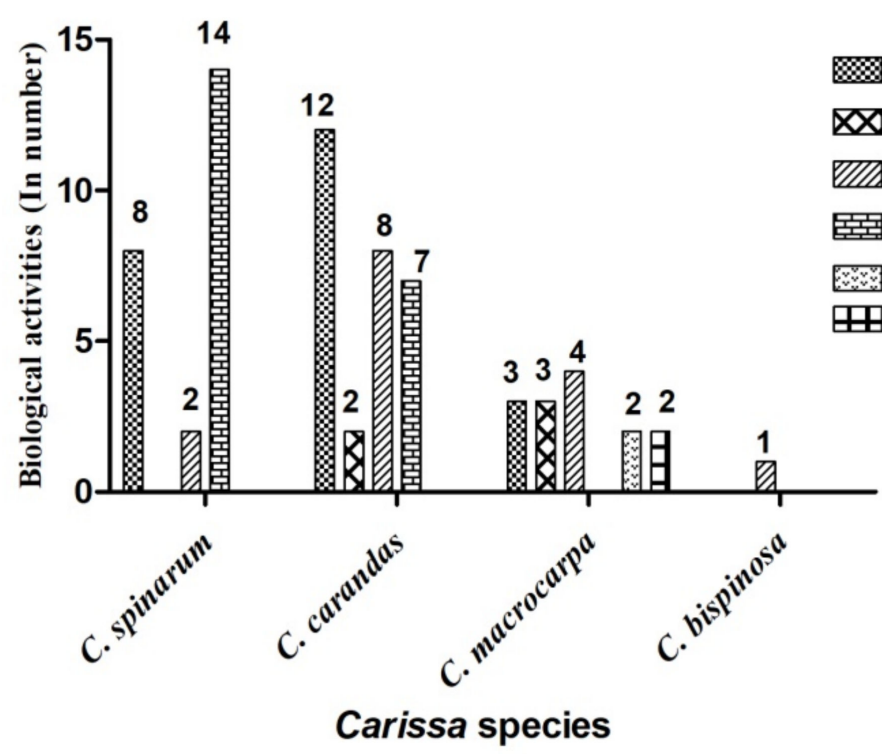

Figure 4. Ratio of biological activities screened during the analysis of the literature from C. spinarum, C. carandas, C. macrocarpa, and C. bispinosa.

This review article concludes that crude extracts from different parts of Carissa species possess significant anti-inflammatory, antiarthritic, adaptogenic, antidiabetic, antimalarial, antiplasmoidal, anticonvulsant, and antiviral activities in in vitro as well as in vivo conditions. However, the mechanism of action of extracts/phytocompounds for their antioxidant, antimicrobial, anticancer, and cardioprotective potential using different model systems is still required. In most of the in vivo studies, the extracts of Carissa species showed significant pharmacological activities (anti-inflammatory, hepatoprotective, antipyretic, antimalarial, and antiviral activities) at a concentration between 100 and $500 \mathrm{mg} / \mathrm{kg}$. Toxicity studies revealed that $C$. spinarum and C. carandas could be used at up to a $5000 \mathrm{mg} / \mathrm{kg}$ dose level without any harmful effect. The various phytochemicals such as alkaloids, phenolic lignins, terpenoids, tannins, coumarins, saponin, and glycosides in the leaves, seed, root, 
stem, or the entire plant of Carissa species are the reason behind all pharmacological activities. These investigations support the traditional use of the genus Carissa to treat several ailments, including inflammation, diabetes, malaria, cold, fever, liver, and heart disease. The richness of the Carissa fruits in antioxidants, vitamin C, and minerals validates their use as a food additive in various food preparations. Although several compounds of Carissa species (C. macrocarpa, C. bispinosa, and C. spinarum) have been isolated, all these compounds are still not explored for their biological potentials. Thus, the study also concludes that C. macrocarpa is the least explored species in terms of extraction of pure compounds. The clinical evaluation of crude extracts and pure compounds from the less explored Carissa species in vivo model is still required. Further, the determination of the mechanism of molecular activity of plant extract and its chemical compounds within animal model systems still needs to be explored. The current study is intended to help researchers to acknowledge the therapeutic potential of all plant species of the Carissa genus.

\section{Future Recommendations}

The present study recommends exploring the unexplored species of the genus Carissa, e.g., C. boiviniana, C. haematocarpa, C. pichoniana, and C. tetramera, for their chemical and pharmacological profile. Further analysis of phytoconstituents from C. bispinosa is required to obtain new bioactive compounds with significant biological applications. Moreover, clinical evaluation and in vivo models of crude extracts and pure compounds of Carissa species are still required to be standardised. Further, the determination of the mechanism of molecular activity of the plant extract and its chemical compounds within animal model systems still needs to be explored.

Author Contributions: Conceptualisation A.K. (Amita Kumari), J.D. and R.A.; Manuscript writing, J.D., I.G., S.L., S.T. and K.G.; Manuscript editing, A.K. (Amita Kumari), R.V., V.K., N.U., J.C.-J.C. and S.S.; Critical revising, A.K. (Ashwani Kumar), J.M.L., R.A. and A.-L.E.M. All authors have read and agreed to the published version of the manuscript.

Funding: This research received no external funding.

Institutional Review Board Statement: Not applicable.

Informed Consent Statement: Not applicable.

Acknowledgments: The authors would like to express their special thanks to Neeraj Pizzar, Assistant Professor cum Assistant Director, Scientific writing cell, Shoolini University, Solan, H.P., India, for editing the manuscript.

Conflicts of Interest: The authors declare no conflict of interest in the publication.

Sample Availability: Not applicable.

$\begin{array}{ll}\text { Abbreviations } \\ \mathrm{EC}_{50} & \text { Half maximal effective concentration } \\ \mathrm{IC}_{50} & \text { Inhibitory concentration required for } 50 \% \text { inhibition } \\ \mathrm{SC}_{50} & \text { Scavenging concentration required for } 50 \% \text { scavenging } \\ \mathrm{GI}_{50} & \text { Growth inhibition } \\ \mathrm{LD}_{50} & \text { Lethal dose } \\ \mathrm{MIC} & \text { Minimum inhibitory concentration } \\ \mathrm{mg} / \mathrm{mL} & \text { Milligrams per millilitre } \\ \mathrm{mg} / \mathrm{kg} & \text { Milligrams per kilogram } \\ \mu \mathrm{M} & \text { Micromolar } \\ \mu \mathrm{g} / \mathrm{mL} & \text { Microgram per millilitre } \\ \mathrm{mol} / \mathrm{min} / \mathrm{g} & \text { Mole per min per gram }\end{array}$




$\begin{array}{ll}\mathrm{g} / \mathrm{kg} & \text { Gram per kilogram } \\ \mathrm{kg} / \mathrm{mL} & \text { Kilogram per millilitre } \\ \text { et al } & \text { et alia } \\ \mathrm{min} . & \text { Minimum } \\ \mathrm{syn} . & \text { Synonym } \\ v / v & \text { Volume per volume }\end{array}$

\section{References}

1. Lindsay, E.A.; Berry, Y.; Jamie, J.F.; Bremner, J.B. Antibacterial compounds from Carissa lanceolata R Br. Phytochemistry 2000, 55, 403-406. [CrossRef]

2. The Plant List. Version 1. Available online: http://www.theplantlist.org (accessed on 1 January 2010).

3. Ngulde, S.I.; Sandabe, U.K.; Tijjani, M.B.; Barkindo, A.A.; Hussaini, I.M. Phytochemical constituents antimicrobial screening and acute toxicity studies of the ethanol extract of Carissa edulis Vahl root bark in rats and mice. Am. J. Res. Commun. 2013, 1, 99-110.

4. Fanta Yadang, S.A.; Taiwe Sotoing, G.; Ngatcha Zouakeu, K.S.; Khan, M.A.; Agbor, G.A.; Ur-Rahman, N.; Ngo Bum, E. Quantification of bioactive compounds and evaluation of the antioxidant activity of Carissa edulis Vahl. (Apocynaceae) leaves. Sci. World J. 2019, 2019, 7549620. [CrossRef] [PubMed]

5. Yau, J.; Yaro, A.H.; Abubakar, M.S.; Anuka, J.A.; Hussaini, I.M. Anticonvulsant activity of Carissa edulis (Vahl) (Apocynaceae) root bark extract. J. Ethnopharmacol. 2008, 120, 255-258. [CrossRef]

6. Dar, M.A.; Kumar, J.; Sami, R. Anti-arthritic activity of leaf of Carissa carandas (L) against adjuvant-induced arthritis in rat. J. Nat. Ayurvedic Med. 2019, 2019, 000187. [CrossRef]

7. Souilem, F.; El Ayeb, A.; Djlassi, B.; Ayari, O.; Chiboub, W.; Arbi, F.; Ascrizzi, R.; Flamini, G.; Harzallah-Skhiri, F. Chemical composition and activity of essential oils of Carissa macrocarpa (Eckl) A DC cultivated in Tunisia and its anatomical features. Chem. Biodivers. 2018, 15, e1800188. [CrossRef]

8. Moodley, R.; Chenia, H.; Jonnalagadda, S.B.; Koorbanally, N. Antibacterial and anti-adhesion activity of the pentacyclic triterpenoids isolated from the leaves and edible fruits of Carissa macrocarpa. J. Med. Plant Res. 2011, 5, 4851-4858.

9. Gwatidzo, L.; Dzomba, P.; Mangena, M. TLC separation and antioxidant activity of flavonoids from Carissa bispinosa, Ficus sycomorus and Grewia bicolar fruits. Nutrire 2018, 43, 1-3. [CrossRef]

10. Ahmed, D.; Waheed, A.; Chaudhary, M.A.; Khan, S.R.; Hannan, A.; Barkaat, M. Nutritional and antimicrobial studies on leaves and fruit of Carissa opaca Stapf ex Haines. Asian J. Chem. 2011, 23, 2072-2076. [CrossRef]

11. Kaunda, J.S.; Zhang, Y.J. The genus Carissa: An ethnopharmacological, phytochemical and pharmacological review. Nat. Prod. Biopros. 2017, 7, 181-199. [CrossRef]

12. Arif, M.; Kamal, M.; Jawaid, T.; Khalid, M.; Saini, K.S.; Kumar, A.; Ahmad, M. Carissa carandas Linn. (Karonda): An exotic minor plant fruit with immense value in nutraceutical and pharmaceutical industries. Asian J. Biomed. Pharmaceut. Sci. 2016, 6, 14-19.

13. Berhanu, G.; Atalel, D.; Kandi, V. A Review of the Medicinal and Antimicrobial Properties of Carissa spinarum L. Am. J. Biomed. Res. 2020, 8, 54-58.

14. POWO. Plants of the world online. Facilitated by the Royal Botanic Garden, Kew. 2019. Available online: http://www. plantoftheworldonline.org/ (accessed on 10 November 2021).

15. Wheeler, J.R.; Rye, B.L.; Koch, B.L.; Wilson, A.J.G. Western Australian Herbarium. In Flora of the Kimberley Region; Western Australian Herbarium Como: Kensington, WA, Australia, 1992; p. 1392.

16. Fatima, A.; Singh, P.P.; Agarwal, P.; Irchhaiya, R.; Alok, S.; Verma, A. Treatment of various diseases by Carissa spinarum L.: A promising shrub. IJPSR. 2013, 4, 2489-2495.

17. Ahmed, D.; Fatima, K.; Saeed, R. Analysis of phenolic and flavonoid contents and the anti-oxidative potential and lipid peroxidation inhibitory activity of methanolic extract of Carissa opaca roots and its fractions in different solvents. Antioxidants 2014, 3, 671-683. [CrossRef]

18. Yilangai, R.M.; Chaskda, A.A.; Mwansat, G.S. Avian utilization of the fruits of Carissa edulis Vahl and Jasminum Dichotomum Vahl in a Central Nigerian Reserve. J. Nat. Sci. Res. 2014, 4, 5-10.

19. Tascioglu, S.; Kuzucu, M. Importance of Natal plum (Carissa grandiflora) as a landscape plant in turkey. Int. J. Eng. Res. Manag. Stud. 2018, 5, 1-6.

20. Parvin, N. Phytochemical screening antinociceptive anthelmintic and cytotoxicity studies of the leaves of Carissa carandas Linn (Family, Apocynaceae). Int. J. Sci. Rep. 2018, 4, 119-123. [CrossRef]

21. Hegde, K.; Thakker, S.P.; Joshi, A.B.; Shastry, C.S.; Chandrashekhar, K.S. Anticonvulsant activity of Carissa carandas Linn root extract in experimental mice. Trop. J. Pharm. Res. 2009, 8, 117-125. [CrossRef]

22. Chandra, S.; Skalani, S.; Kandari, S.A. Nutritional evaluation antimicrobial activity and phytochemical screening of wild edible fruit (Carissa opaca). Int. Res. J. Pharm. 2011, 2, 217-221.

23. Wangteeraprasert, R.; Lipipun, V.; Gunaratnam, M.; Neidle, S.; Gibbons, S.; Likhitwitayawuid, K. Bioactive compounds from Carissa spinarum. Phytother. Res. 2012, 26, 1496-1499. [CrossRef]

24. Saxena, K.; Irchhaiya, R.; Chagti, K.K. Analytical and medicinal properties of ethanolic extract of leaves of Carissa carandas. World J. Pharm. Sci. 2016, 5, 1683-1690. 
25. Feyissa, D.; Melaku, Y. Phytochemical antibacterial and antioxidant studies of the leaves of Carissa spinarum. Int. J. Pharm. Chem. Sci. 2016, 7, 25-30.

26. Irvine, F.R. Woody Plants of Ghana; Oxford University Press: London, UK, 1961.

27. Sofowora, A. The present status of knowledge of the plants used in traditional medicine in Western Africa: A medical approach and a chemical evaluation. J. Ethnopharmacol. 1980, 2, 109-118. [CrossRef]

28. Burkill, H.M. The Useful Plants of West Tropical Africa; Families AD (No. Ed 2); Royal Botanic Garden: Kew, UK, $1985 ;$ Volume 1.

29. Omino, E.A.; Kokwaro, J.O. Ethnobotany of Apocynaceae species in Kenya. J. Ethnopharmacol. 1993, 40, 167-180. [CrossRef]

30. Oliver, B. Medicinal Plants in Nigeria; The Nigerian College of Arts Science and Technology: Lagos, Nigeria, $1994 ;$ Volume 52.

31. Ibrahim, H.; Oyi, R.A.; Ehinmidu, J.O.; Musa, K.Y.; Bright, N.T. Antimicrobial activity of the water extracts of the leaves and fruits of Carissa edulis Vahl (Apocynaceae). J. Med. Plant Res. 2010, 4, 1028-1032. [CrossRef]

32. Chopra, R.N.; Nayar, S.L.; Chopra, I.C. Glossary of Medicinal Plants of India; CSIR: New Delhi, India, 1956.

33. Reid, E.J.; Betts, T.J. The Record of Western Australian Plants Used by Aboriginals as Medicinal Agents; Perth, Curtin University of Technology: Bentley, WA, Australian, 1979.

34. Awasthi, A.K.; Kunal, K.; Bisht, G.S.; Awasthi, S. In vitro antibacterial and antifungal activity of Carissa opaca Stapf ex Haines. Int. J. Curr. Pharm. Res. 2013, 5, 15-18.

35. Ahmed, D.; Saeed, R.; Shakeel, N.; Fatima, K.; Arshad, A. Antimicrobial activities of methanolic extract of Carissa opaca roots and its fractions and compounds isolated from the most active ethyl acetate fraction. Asian Pac. J. Trop. Biomed. 2015, 5, 541-545. [CrossRef]

36. Chauhan, A.M.; Tanwar, B.E.; Arneja, I.N. Influence of processing on physiochemical nutritional and phytochemical composition of Carissa spinarum (karonda) fruit. Asian J. Pharm. Clin. Res. 2015, 1, 254-259.

37. Harwansh, R.K.; Garabadu, D.; Rahman, M.A.; Garabadu, P.S. In-vitro anthelmintic activity of different extracts of root of Carissa Spinarum. Int. J. Pharma. Sci. Res. 2010, 8, 81-84.

38. Jayaweera, D.M.A. Medicinal Plants (Indigenous and Exotic) Used in Ceylon: Flacourtiaceae- Lythraceae; National Science Council of Sri Lanka: Colombo, Sri Lanka, 1981.

39. Hettiarachchi, D.S.; Locher, C.; Longmore, R.B. Antibacterial compounds from the root of the indigenous Australian medicinal plant Carissa lanceolata R Br. Nat. Prod. Res. 2011, 25, 1388-1395. [CrossRef]

40. Sastri, B.N. The Wealth of India New Delhi; Publications and Information Directorate, CSIR: New Delhi, India, 1950.

41. Begum, S.; Syed, S.A.; Siddiqui, B.S.; Sattar, S.A.; Choudhary, M.I. Carandinol: First isohopane triterpene from the leaves of Carissa carandas L. and its cytotoxicity against cancer cell lines. Phytochem. Lett. 2013, 6, 91-95. [CrossRef]

42. Anupama, N.; Madhumitha, G.; Rajesh, K.S. Role of dried fruits of Carissa carandas as anti-inflammatory agents and the analysis of phytochemical constituents by GC-MS. Biomed. Res. Int. 2014, 2014, 512369. [CrossRef] [PubMed]

43. Kirtikar, K.R.; Basu, B.D. Indian Medicinal Plant, 2nd ed.; CABI: Allahabad, India, 2003.

44. Bint-e-Sadek, Y.; Choudhury, N.; Shahriar, M. Biological investigations of the leaf extracts of Carissa carandas. Int. J. Pharm. Res. Technol. 2013, 5, 97-105.

45. The National Academies. Lost Crops of Africa: Volume III, Fruits; The National Academies Press: Washington, DC, USA, 2008. [CrossRef]

46. Souilem, F.; Dias, M.I.; Barros, L.; Calhelha, R.C.; Alves, M.J.; Harzallah-Skhiri, F.; Ferreira, I.C. Amantagula fruit (Carissa macrocarpa (Eckl) A DC): Nutritional and phytochemical characterization. Plant Foods for Hum. Nutr. 2019, 74, 76-82. [CrossRef]

47. Swart, W.J. Survival Off the Bush-5: The Yummy Num-Nums. Farmer's Weekly, 29 October 1982; 58-59.

48. Hegde, K.; Joshi, A.B. Hepatoprotective effect of Carissa carandas Linn root extract against $\mathrm{CCl}_{4}$ and paracetamol induced hepatic oxidative stress. Indian J. Exp. Biol. 2009, 47, 660-667.

49. Jayakumar, K.; Muthuraman, B. Traditional uses and nutrient status of Indian native plant fruit (Carissa carandas Linn). World Sci. News 2018, 96, 217-224.

50. Musinguzi, E.; Kikafunda, J.K.; Kiremire, B.T. Promoting indigenous wild edible fruits to complement roots and tuber crops in alleviating vitamin A deficiency in Uganda. Proc. 13th ISTRC Symp. 2007, 763-769.

51. Krishna, H.; Chauhan, N.; Sharma, B.D. Evaluation of Karonda (Carissa carandas L.) derived natural colourant cum nutraceuticalssupplement. IJMFM AP 2007, 3, 28-33.

52. Sarma, A.; Sarmah, P.; Kashyap, D.; Dutta, S.; Mahanta, M. Antioxidant activity and nutraceutical property of the fruits of an ethno-medicinal plant, Carissa carandas L found in Brahmaputra valley agro-climatic condition. J. Pharm. Sci. Res. 2015, 7, 55-57.

53. Muhammad, A.; Sheeba, F. Pharmacognostical and preliminary phytochemical analysis of Carissa carandas fruits. J. Med. Aromat. Plants Sci. 2011, 33, 53-58.

54. Dalal, R.P.; Thakur, N.A.; Singh, A. Nutritional value of Karonda (Carissa carandas Linn). A non-conventional fruit under semi-arid condition of Punjab. Indian J. Agrofor. 2010, 12, 102-104.

55. Sarkar, R.; Kundu, A.; Banerjee, K.; Saha, S. Anthocyanin composition and potential bioactivity of Karonda (Carissa carandas L) fruit: An Indian source of biocolorant. LWT Food Sci. Tech. 2018, 93, 673-678. [CrossRef]

56. Wangteeraprasert, R.; Likhitwitayawuid, K. Lignans and a sesquiterpene glucoside from Carissa carandas stem. Helv. Chim. Acta 2009, 92, 1217-1223. [CrossRef]

57. Hegde, K.; Joshi, A.B. Preliminary phytochemical screening and antipyretic activity of Carissa spinarum root extract. Pharm. Lett. 2010, 2, 255-260.

58. Sahreen, S.; Khan, M.R.; Khan, R.A. Hepatoprotective effects of methanol extract of Carissa opaca leaves on $\mathrm{CCl}_{4}$-induced damage in rat. BMC Complement. Altern. Med. 2011,11, 48. [CrossRef] 
59. Verma, S.; Chaudhary, H.S. Effect of Carissa carandas against clinically pathogenic bacterial strains. J. Pharm. Res. 2011, 4, 3769-3771.

60. Nisa, S.; Bibi, Y.; Zia, M. Anticancer investigations on Carissa opaca and Toona ciliata extracts against human breast carcinoma cell line. Pak. J. Pharm. Sci. 2013, 26, 1009-1012.

61. Mishra, C.K.; Shrivastava, B.; Sasmal, D. Pharmacognostical standardization and phytochemical identification of fruit and root of Carissa carandas linn. Int. J. Pharm. Sci. 2013, 5, 347-350.

62. Onotu, C.S.; Musa, U.B.; Fajinmi, A.O.; Mazadu, M.R.; Shaida, S.S. Physiochemical evaluation of ethanolic root extract of Carissa Spinarum (Wild Karanda) on Trypanosoma Brucei (Federe Strain) infected mice. Int. J. Pharm. Sci. 2013, 2, 18-26.

63. Anupama, N.; Madhumitha, G. Pharmacognostic standardization and anti-microbial studies of dried Carissa carandas fruits. Int. J. Pharm. Tech. Res. 2015, 8, 206-210.

64. Mishra, C.K.; Sasmal, D. In-vivo evaluation of anti-diarrhoeal activity of ethanolic fruit and root extracts of Carissa carandas Linn (Apocynaceae). Int. J. Drug Dev. Res. 2015, 7, 216-221.

65. Jorum, O.H.; Piero, N.M.; Machocho, A.K. Hematological effects of dichloromethane-methanolic leaf extracts of Carissa edulis (Forssk) Vahl in normal rat models. J. Hemat. Thromb. Dis. 2016, 4, 2-3.

66. Kumar, V.; Tarpada, P.; Sadariya, K.; Goswami, S. Comparative phytochemical and antioxidant activities of methanol and petroleum ether extract of Carissa carandas leaves fruit and seed. Vivechan Int. J. Res. 2017, 8, 70-75.

67. Raina, M.K.; Bhatnagar, J.K.; Atal, C.K. Isolation of caffeic acid from the roots of Carissa spinarum L. Indian J. Pharm. 1971, 33, 76-77.

68. Al-Youssef, H.M.; Hassan, W.H. Phytochemical and biological studies of the aerial parts of Carissa edulis growing in Saudi Arabia. Biomed. Pharmacol. J. 2012, 5, 9-18. [CrossRef]

69. Al-Youssef, H.M.; Hassan, W.H. Chemical constituents of Carissa edulis Vahl. Arab. J. Chem. 2017, 10, 109-113. [CrossRef]

70. El-Desoky, A.H.; Abdel-Rahman, R.F.; Ahmed, O.K.; El-Beltagi, H.S.; Hattori, M. Anti-inflammatory and antioxidant activities of naringin isolated from Carissa carandas L.: In vitro and in-vivo evidence. Phytomedicine 2018, 42, 126-134. [CrossRef]

71. Khalil, H.E.; Mohamed, M.E.; Morsy, M.A.; Kandeel, M. Flavonoid and phenolic compounds from Carissa macrocarpa: Molecular docking and cytotoxicity studies. Pharmacogn. Mag. 2018, 14, 304. [CrossRef]

72. Parveen, S.; Riaz, N.; Saleem, M.; Ashraf, M.; Khatoon, T.; Nisar, M.F.; Jabbar, A. A new cyclic terephthalate from the methanolic extract of Carissa opaca. Chem. Soc. Pak. 2017, 39, 610-613.

73. Liu, Y.; Zhang, Y.; Muema, F.W.; Kimutai, F.; Chen, G.; Guo, M. Phenolic Compounds from Carissa spinarum are characterized by their antioxidant, anti-inflammatory and hepatoprotective activities. Antioxidants 2021, 10, 652. [CrossRef] [PubMed]

74. Sahreen, S.; Khan, M.R.; Khan, R.A. Evaluation of antioxidant activities of various solvent extracts of Carissa opaca fruits. Food Chem. 2010, 122, 1205-1211. [CrossRef]

75. Sahreen, S.; Khan, M.R.; Khan, R.A. Evaluation of antioxidant profile of various solvent extracts of Carissa opaca leaves: An edible plant. Chem. Cent. J. 2017, 11, 83. [CrossRef] [PubMed]

76. Ahmed, D.; Hammad, M. Comparative study of antioxidant antimicrobial and a-amylase inhibitory activities of leaves fruits and seeds of Carissa opaca. Asian J. Chem. 2013, 25, 3913-3916. [CrossRef]

77. Fowsiya, J.; Madhumitha, G. Preliminary phytochemical analysis antioxidant and cytotoxicity test of Carissa edulis Vahl dried fruits. IOP Conf. Ser. Mater. Sci. Eng. 2017, 263, 022018. [CrossRef]

78. Rao, R.J.; Kumar, S.U.; Reddy, V.S.; Tiwari, A.K.; Rao, J. Antioxidants and a new germacrane sesquiterpene from Carissa spinarum. Nat. Prod. Res. 2005, 19, 763-769. [CrossRef]

79. Bhadane, B.S.; Patil, R.H. Isolation purification and characterization of antioxidative steroid derivative from methanolic extract of Carissa carandas (L) leaves. Biocatal. Agric. Biotechnol. 2017, 10, 216-223. [CrossRef]

80. Khatun, M.; Habib, M.R.; Rabbi, M.A.; Amin, R.; Islam, M.F.; Nurujjaman, M.; Karim, M.R.; Rahman, M.H. Antioxidant cytotoxic and antineoplastic effects of Carissa carandas Linn leaves. Exp. Toxicol. Pathol. 2017, 69, 469-476. [CrossRef]

81. Verma, K.; Shrivastava, D.; Kumar, G. Antioxidant activity and DNA damage inhibition in vitro by a methanolic extract of Carissa carandas (Apocynaceae) leaves. J. Taibah Univ. Sci. 2015, 9, 34-40. [CrossRef]

82. Mishra, C.K.; Sasmal, D.; Kumar, D. In vitro Antioxidant activity of chloroform and ethanolic fruit and root extracts of Carissa carandas Linn. J. Innov. Pharm. Sci. 2017, 1, 41-45.

83. Souilem, F.; Dias, M.I.; Barros, L.; Calhelha, R.C.; Alves, M.J.; Harzallah-Skhiri, F.; Ferreira, I.C. Phenolic profile and bioactive properties of Carissa macrocarpa (Eckl) A DC: An in-vitro comparative study between leaves stems and flowers. Molecules 2019, 24, 1696. [CrossRef]

84. Hegde, K.; Joshi, A.B. Phytochemical investigation of root extract of the plant Carissa carandas Linn. Res. J. Pharm. Technol. 2010, 3, 217-220.

85. Rumjuankiat, K.; Sonhom, N.; Showpanish, K.; Somsri, A.; Pilasombut, K. In vitro antioxidant activities and volatile compounds from Karanda (Carissa carandas L) fruit wine. Int. J. Agric. Res. 2018, 14, 1843-1860.

86. Rubaka, C.; Ndakidemi, P.; Alebo, H.M.; Shahad, F. Individual and combined antibacterial activity of crude extracts from medicinal plants Carissa spinarum Linn and Carica papaya Linn. Eur. J. Med. Plants 2014, 22, 1513-1523. [CrossRef]

87. David, M.; Karekalammanavar, G. Spectrographic analysis and in vitro study of antibacterial anticancer activity of aqueous ethanolic fruit extract of Carissa carandas. J. Adv. Sci. Res. 2015, 6, 10-13.

88. Fartyal, M.; Kumar, P. Bioactivity of crude extracts of Carissa carandas Linn extracted in polar and non-polar solvents. Int. J. Sci. Res. Innov. Stud. 2014, 3, 186-191. 
89. Abbas, M.; Rasool, N.; Riaz, M.; Zubair, M.; Abbas, M.; Noor-Ul-Haq; Hayat, N. GC-MS profiling, antioxidant, and antimicrobial studies of various parts of Carissa grandiflora. Bulg. Chem. Commun. 2014, 46, 831-839.

90. Gupta, P.; Bhatnagar, I.; Kim, S.K.; Verma, A.K.; Sharma, A. In-vitro cancer cell cytotoxicity and alpha amylase inhibition effect of seven tropical fruit residues. Asian Pac. J. Trop. Biomed. 2014, 4, S665-S671. [CrossRef]

91. Pathak, G.; Singh, S.; Singhal, M.; Singh, J.; Hussain, Y.; Gupta, M.; Meena, A.; Gupta, P.; Rout, P.K. Pharmacology of Carissa carandas leaf extract: Anti-proliferative, Antioxidant and Antimicrobial investigation. Plant Biosyst. Int. J. Deal. All Asp. Plant Biol. 2020, 155, 543-556. [CrossRef]

92. Alqathama, A.; Bader, A.; Khondkar, P.; Gibbons, S.; Prieto, J. Bio guided isolation of cytotoxic compounds against melanoma cells from Carissa spinarum L. Planta Med. 2015, 81, 51-55. [CrossRef]

93. Sehar, I.; Pal, H.C.; Shukla, S.; Bhushan, S.; Hamid, A.; Gupta, B.D.; Saxena, A.K. Cytotoxic evaluation and induction of mitochondria-mediated apoptosis in human leukaemia HL-60 cells by Carissa spinarum stem isolate. J. Pharm. Pharmacol. 2011, 63, 1078-1090. [CrossRef]

94. Kaunda, J.S.; Lokhande, S.J.; Verma, P.R.; Arora, S.K.; Sahu, R.A.; Patil, A.T. Ten new glycosides, carissaedulosides A-J from the root barks of Carissa edulis and their cytotoxicities. Bioorg. Chem. 2020, 102, 104097. [CrossRef]

95. Bano, Z.; Begum, S.; Ali, S.S.; Kiran, Z.; Siddiqui, B.S.; Khawaja, S.; Ahmed, A. Novel ursane-type triterpene from the fresh leaves of Carissa carandas and evaluation of cytotoxicity. J. Asian Nat. Prod. Res. 2020, 9, 892-898. [CrossRef]

96. Sharma, V.; Heer, A.; Kour, N.; Sharma, A.; Singh, S.K. Karonda and Jamun seeds' in vitro anticancer efficacy. Indian J. Tradit. Knowl. 2019, 18, 573-578.

97. Sulaiman, S.F.; Wong, S.T.; Ooi, K.L.; Yusof, S.R.; Muhammad, T.; Sifzizul, T. Anticancer study of Carissa carandas extracts. Project Report, Monograph Universiti, Sains Malaysia. 2008. Available online: http:/ / eprints.usm.my/180/1/Anticancer_study_of_ Carissa_carandas_extracts.pdf (accessed on 10 November 2021).

98. Doshi, G.M. In vitro cytotoxicity studies on Carissa congesta, Polyalthia longifolia, and Benincasa hispida extracts by Sulforhodamine B assay method. Int. J. Green Pharm. 2015, 9, 157-161. [CrossRef]

99. Achenbach, H.; Waibel, R.; Addae-Mensah, I. Lignans and other constituents from Carissa edulis. Phytochemistry 1983, 22, 749-753. [CrossRef]

100. Zafar, N.; Khan, M.A.; Nizami, S.S. Isolation of a new triterpenic alcohol from Carissa carandas. Pak. J. Sci. Ind. Res. 1985, $28,378-381$.

101. Achenbach, H.; Waibel, R.; Addae-Mensah, I. Sesquiterpenes from Carissa edulis. Phytochemistry 1985, 24, 2325-2328. [CrossRef]

102. Naim, Z.; Khan, M.A.; Nizami, S.S. Isolation of a new isomer of ursolic acid from fruits and leaves of Carissa carandas. Pak. J. Sci. Ind. Res. 1988, 31, 753-755.

103. Siddiqui, B.S.; Ghani, U.; Ali, S.T.; Usmani, S.B.; Begum, S. Triterpenoidal constituents of the leaves of Carissa carandas. Nat. Prod. Res. 2003, 17, 153-158. [CrossRef] [PubMed]

104. Karunakar, H.; Thakker, S.P.; Joshi, A.B. Isolation and characterization of chemical constituents from the roots of Carissa carandas. Asian J. Chem. 2009, 21, 5399-5402.

105. Hegde, K.; Issac, C.; Joshi, A. Antiarthritic activity of Carissa spinarum root extract in freund's adjuvant induced polyarthritis in rats. Pharmacol. Online 2010, 2, 713-718.

106. Tolo, F.M.; Rukunga, G.W.; Muli, F.W.; Ochora, J.M.; Irungu, B.N.; Muthaura, C.N.; Wanjiku, C.K.; Mungai, G.M.; Ngoc, Q.; Hashimoto, K.; et al. The antiviral activity of compounds isolated from Kenyan Carissa edulis (Forssk) Vahl. J. Med. Plant Res. 2010, 4, 1517-1522. [CrossRef]

107. Joshi, D.; Boyce, S. Chemical investigation of roots of Carissa congesta, Santapau. I. Isolation of Carissone and D-Glucoside of ß-Sisterol. J. Org. Chem. 1957, 22, 95-97. [CrossRef]

108. Karunakar, H.; Satyanarayana, D.; Joshi, A.B. Phytochemical investigation of root extract of the plant Carissa spinarum. Rajiv Gandhi Univ. Health Sci. J. Pharm. Sci. 2012, 2, 55-59.

109. Arif, M.; Fareed, S.; Hussain, T.; Ali, M. Adaptogenic activity of lanostane triterpenoid isolated from Carissa carandas fruit against physically and chemically challenged experimental mice. Pharmacognosy 2013, 5, 216-220. [CrossRef]

110. Galipalli, S.; Patel, N.K.; Prasanna, K.; Bhutani, K.K. Activity-guided investigation of Carissa carandas (L.) roots for antiinflammatory constituents. Nat. Prod. Res. 2015, 29, 1670-1672. [CrossRef]

111. Parveen, S.; Saleem, M.; Riaz, N.; Ashraf, M.; Nisar, Q.U.M.F.; Jabbar, A. new norterpenoids and a sphingolipid from Carissa opaca. J. Asian Nat. Prod. Res. 2016, 18, 222-231. [CrossRef]

112. Parveen, S.; Riaz, N.; Saleem, M.; Ashraf, M.; Khatoon, T.; Nisar, F.; Jabbar, A. Noropacursane, A new nortriterpenoid from the methanolic extract of Carissa opaca. Rec. Nat. Prod. 2018, 12, 512-517. [CrossRef]

113. Terry, P.; Terry, J.B.; Wolk, A. Fruit and vegetable consumption in the prevention of cancer: An update. J. Intern. Med. 2001, 250, 280-290. [CrossRef]

114. Liyana-Pathirana, C.; Dexter, J.; Shahidi, F. Antioxidant properties of wheat as affected by pearling. J. Agric. Food Chem. 2006, 54, 6177-6184. [CrossRef]

115. Dutta, D.; Nath, A.; Mishra, D.; Verma, N.; Kumar, P. Phytochemical studies on antioxidant activities of two types of Karonda (Carissa carandas) during storage. Ind. J. Hortic. 2016, 73, 623-626. [CrossRef]

116. Rios, J.L.; Recio, M.C.; Villar, A. Screening methods for natural products with antimicrobial activity, a review of the literature. J. Ethnopharmacol. 1988, 23, 127-149. [CrossRef]

117. Andrews, J.M. BSAC standardized disc susceptibility testing method. J. Antimicrob. Chemother. 2001, 48, 43-57. [CrossRef] 
118. Kebenei, J.S.; Ndalut, P.K.; Sabah, A.O. Anti-plasmodial activity of Nortrachelogenin from the root bark of Carissa edulis (Vahl). Int. J. Appl. Res. Nat. Prod. 2011, 4, 1-5.

119. Bapna, S.; Ramaiya, M.; Chowdhary, A. Antimalarial activity of Carissa carandas Linn against Plasmodium falciparum. Photon J. Microbiol. 2013, 12, 246-250.

120. Gebrehiwot, S.; Giday, M.; Erko, B.; Mekonnen, Y. Evaluation of antimalarial activity of a traditionally used medicinal plant in Ethiopia against Plasmodium berghei in Swiss Albino Mice. Int. J. Pharm. Biol. Sci. 2019, 14, 70-76.

121. Gertsch, J. How scientific is the science in ethnopharmacology? Historical perspectives and epistemological problems. J. Ethnopharmacol. 2009, 122, 177-183. [CrossRef] [PubMed]

122. Tolo, F.M.; Rukunga, G.M.; Muli, F.W.; Njagi, E.N.; Njue, W.; Kumon, K.; Mungai, G.M.; Muthaura, C.N.; Muli, J.M.; Keter, L.K.; et al. Anti-viral activity of the extracts of a Kenyan medicinal plant Carissa edulis against herpes simplex virus. J. Ethnopharmacol. 2006, 104, 92-99. [CrossRef] [PubMed]

123. Islam, M.R.; Rahman, S.M.; Ahmed, M.; Das, P.R.; Tabibul, M.; Islam, M.H.; Ahmad, I.; Rahmatullah, M. Antinociceptive activity studies with methanol extract of Annona reticulata L. (Annonaceae) and Carissa carandas L (Apocynaceae) leaves in Swiss albino mice. Adv. Nat. Appl. Sci. 2012, 6, 1313-1318.

124. Jamilu, Y.; Yaro, A.H.; Abubakar, M.S.; Hussaini, I.M.; Anuka, J.A. Studies on anticonvulsant activity of fractions of hydro-alcoholic root bark extract of Carissa edulis (Vahl). Niger. J. Pharm. Sci. 2007, 6, 61-66.

125. Maina, G.S.; Kelvin, J.K.; Maina, M.B.; Muriithi, N.J.; Kiambi, M.J.; Umar, A.; John, M.K.; Ann, N.W.; David, M.N.; Piero, N.M. Antinociceptive properties of dichloromethane: Methanolic leaf and root bark extracts of Carissa edulis in rats. J. Phytopharm. 2015, $4,106-112$.

126. Mworia, J.K.; Gitahi, S.M.; Juma, K.K.; Njagi, J.M.; Mwangi, B.M.; Aliyu, U. Antinociceptive activities of acetone leaves extracts of Carissa Spinarum in Mice. J. Med. Aromat. Plants 2015, S1, 006.

127. El-Fiky, F.K.; Abou-Karam, M.A.; Afify, E.A. Effect of Luffa aegyptiaca (seeds) and Carissa edulis (leaves) extracts on blood glucose level of normal and streptozotocin diabetic rats. J. Ethnopharmacol. 1996, 50, 43-47. [CrossRef]

128. Swami, G.; Nagpal, N.; Rahar, S.; Preeti, S.; Porwal, A.; Nagpal, A.M.; Kapoor, R. Effect of aqueous leaves extract of Carissa carandas Linn on blood glucose levels of normoglycemic \& alloxan induced diabetic Wister rats. Int. J. Curr. Pharm. Res. 2010, 2, 65-67.

129. Itankar, P.R.; Lokhande, S.J.; Verma, P.R.; Arora, S.K.; Sahu, R.A.; Patil, A.T. Antidiabetic potential of unripe Carissa carandas Linn fruit extract. J. Ethnopharmacol. 2011, 135, 430-433. [CrossRef]

130. Madhuri, S.; Neelagund, S.E. Anti-oxidant anti-diabetic activity and DNA damage inhibition activity of Carissa carandas fruit. Int. J. Adv. Res. Dev. 2019, 4, 75-82.

131. Garg, V.K.; Paliwal, S.K.; Sharma, S. Analgesic and antipyretic activities of aqueous extract of leaves of Carissa carandas Linn. Pharmacol. Online 2011, 1, 1109-1119.

132. Hati, M.; Jena, B.K.; Kar, S.; Nayak, A.K. Evaluation of anti-inflammatory and anti-pyretic activity of Carissa carandas L. leaf extract in rats. Int. J. Pharm. Chem. Bio. Sci. 2014, 1, 18-25.

133. Bhaskar, V.H.; Balakrishnan, N. Analgesic anti-inflammatory and antipyretic activities of Pergularia daemia and Carissa carandas. Daru J. Pharm. Sci. 2009, 17, 168-174.

134. Gitahi, S.M. Antipyretic properties of dichloromethane: Methanolic leaf and root bark extracts of Carissa edulis in rats. Asian J. Biomed. Pharm. 2015, 5, 12-20.

135. Allam, K.M.; El-kader, A.; Adel, M.; Fouad, M.A.; Mostafa, M.A. Phytochemical and Biological Studies of Carissa macrocarpa, F. Apocyanaceae. JABPS 2021, 4, 56-64. [CrossRef]

136. Beck, N.R.; Namdeo, K.P. Evaluation of in-vivo Anti-inflammatory activity of leaves extract of Carissa spinarum on formalin induced paw edema in albino rats. J. Pharm. Biosci. 2016, 30, 36-40.

137. Yau, J.; Malami, S.; Abdullahi, S.B.; Yaro, A.H. Analgesic and anti-inflammatory activities of the residual aqueous fraction of Carissa edulis root bark (Vahl) in experimental animals. Afr. J. Pharm. Pharmacol. 2017, 6, 173-177.

138. Woode, E.; Ansah, C.; Ainooson, G.K.; Abotsi, W.M.; Mensah, A.Y.; Duweijua, M. Anti-inflammatory and antioxidant properties of the root extract of Carissa edulis (Forsk) Vahl (Apocynaceae). J. Sci. Technol. 2007, 27, 6-15. [CrossRef]

139. Saher, S.; Narnawre, S.; Patil, J. Evaluation of phytochemical and pharmacological activity of Carissa carandas L. fruits at three different stages of maturation. Drug Res. 2020, 70, 80-85. [CrossRef]

140. Bhaskar, V.H.; Balakrishnan, N. Hepatoprotective activity of laticiferous plant species (Pergularia daemia and Carissa carandas) from Western Ghats Tamilnadu, India. Pharm. Lett. 2009, 1, 130-142.

141. Hegde, K.; Joshi, A.B. Hepatoprotective and anti-oxidant effect of Carissa spinarum root extract against $\mathrm{CCl}_{4}$-and paracetamolinduced hepatic damage in rats. Bangladesh J. Pharm. 2010, 5, 73-76. [CrossRef]

142. John, P.P.; Mazumder, A.; Mazumder, R.; Bhatnagar, S.P. Anthelmintic activity of root bark of Carissa carandas. Anc. Sci. Life 2007, $27,11-13$.

143. Sahreen, S.; Khan, M.R.; Khan, R.A.; Alkreathy, H.M. Cardioprotective role of leaves extracts of Carissa opaca against $\mathrm{CCl}_{4}$ induced toxicity in rats. BMC Res. Notes 2014, 7, 224. [CrossRef]

144. Mishra, C.K.; Sasmal, D.; Shrivastava, B. An in vitro evaluation of the anthelmintic activity of unripe fruits extract of Carissa carandas Linn. Int. J. Drug Dev. Res. 2012, 4, 393-397. 
145. Mohtasheemul, H.M.; Salman, A.; Ziauddin, A.; Iqbal, A. Antiemetic activity of some aromatic plants. J. Pharm. Sci. Innov. 2012, 1, 47-49.

146. Saha, R.; Hossain, L.; Bose, U.; Rahman, A.A. Neuropharmacological and diuretic activities of Carissa carandas Linn leaf. Pharmacol. Online 2010, 2010, 320-327.

147. Yadang, F.S.A.; Nguezeye, Y.; Kom, C.W.; Betote, P.H.D.; Mamat, A.; Tchokouaha, L.R.Y.; Taiwé, G.S.; Agbor, G.A.; Bum, E.N. Scopolamine-Induced Memory Impairment in Mice: Neuroprotective Effects of Carissa edulis (Forssk.) Valh (Apocynaceae) Aqueous Extract. Int. J. Alzheimers Dis. 2020, 2020, 6372059. [CrossRef]

148. Nedi, T.; Mekonnen, N.; Urga, K. Diuretic effect of the crude extracts of Carissa edulis in rats. J. Ethnopharmacol. 2004, 95, 57-61. [CrossRef]

149. Kebamo, S.; Makonnen, E.; Debella, A.; Geleta, B. Evaluation of diuretic activity of different solvent fractions of methanol extract of Carissa edulis root bark in rats. Med. Chem. 2015, 5, 472-478. [CrossRef]

150. Sanwal, R.; Chaudhary, A.K. Wound healing and antimicrobial potential of Carissa spinarum Linn in albino mice. J. Ethnopharmacol. 2011, 135, 792-796. [CrossRef]

151. Gebrehiwot, S. In-vivo acute and sub-acute toxicity study of root extract of Carissa spinarum Linn in Swiss albino mice. Int. J. Pharm. Pharm. Sci. 2019, 11, 62-65. [CrossRef]

152. Shamim, S. Acute subacute and subchronic toxicological studies of Carissa carandas leaves (ethanol extract): A plant active against cardiovascular diseases. J. Dow Univ. Health Sci. 2014, 8, 121-125.

153. Kennedy, G.L.; Ferenz, R.L.; Burgess, B.A. Estimation of acute oral toxicity in rates by determination of the approximate lethal dose rather than the LD50. J. Appl. Toxicol. 1986, 6, 145-148. [CrossRef]

154. Kifayatullah, M.; Mustafa, M.S.; Sengupta, P.; Sarker, M.M.R.; Das, A.; Das, S.K. Evaluation of the acute and sub-acute toxicity of the ethanolic extract of Pericampylus glaucus (Lam.) Merr. in BALB/c mice. J. Acute Dis. 2015, 4, 309-315. [CrossRef]

155. Dossou-Yovo, K.M.; Diallo, A.; Lawson-Evi, P.; Darre, T.; Bakoma, B.; Eklu-Gadegbeku, K. Cytotoxicity, acute and subacute study of hydroalcoholic root extract of Carissa spinarum L. on Wistar rats. J. Med. Food 2021, 24, 756-761. [CrossRef] 